\title{
Decision Support Methods and Tools
}

\author{
Lawrence L. Green ${ }^{1}$, Natalia M. Alexandrov ${ }^{2}$, Sherilyn A. Brown ${ }^{3}$, \\ Jeffrey A. Cerro ${ }^{4}$, Clyde R. Gumbert ${ }^{5}$, and Michael R. Sorokach ${ }^{6}$ \\ NASA Langley Research Center, Hampton, VA 23681 \\ Cécile M. Burg ${ }^{7}$ \\ National Institute of Aerospace, Hampton, VA 23666 \\ Georgia Institute of Technology, Atlanta, GA 30332
}

This paper is one of a set of papers, developed simultaneously and presented within a single conference session, that are intended to highlight systems analysis and design capabilities within the Systems Analysis and Concepts Directorate (SACD) of the National Aeronautics and Space Administration (NASA) Langley Research Center (LaRC). This paper focuses on the specific capabilities of uncertainty/risk analysis, quantification, propagation, decomposition, and management, robust/reliability design methods, and extensions of these capabilities into decision analysis methods within SACD. These disciplines are discussed together herein under the name of Decision Support Methods and Tools. Several examples are discussed which highlight the application of these methods within current or recent aerospace research at the NASA LaRC. Where applicable, commercially available, or government developed software tools are also discussed.

\section{Nomenclature}

$C A I B=$ Columbia Accident Investigation Board

$C D F=$ Cumulative Distribution Function

$D S \quad=$ Decision Support

LaRC = NASA Langley Research Center

NASA $=$ National Aeronautics and Space Administration

$P D F \quad=$ Probability Density Function

$S A \quad=$ Systems Analysis

$S A C D=$ Systems Analysis and Concepts Directorate

$S E \quad=\quad$ Systems Engineering

\section{Introduction}

$\mathrm{T}$

HE discipline of Systems Analysis (SA) at the National Aeronautics and Space Administration (NASA) Langley Research Center (LaRC) encompasses a broad spectrum of analysis and design capabilities devoted to aerospace components and vehicles. These capabilities include a wide variety of traditional vehicle analysis specialty areas (disciplines) such as aerodynamics, structures, controls, heat transfer, and trajectory analysis. But a growing number of people within NASA, and particularly at NASA LaRC, also perform analyses which span across, and are distinct from, these specialty areas to support the process of making decisions based upon computational simulations. For example, systems integration, multidisciplinary optimization, mission and trade space analyses, life cycle cost analyses, uncertainty/risk analysis and management, robust and reliability design methods, technology assessments, research portfolio analyses, and "system of systems" architecture analyses all fall into this category of

\footnotetext{
${ }^{1}$ Aerospace Engineer, Space Mission Analysis Branch, Mail Stop 462, Senior Member of AIAA.

${ }^{2}$ Mathematician, Aeronautics Systems Analysis Branch, Mail Stop 442.

${ }^{3}$ Aerospace Engineer, Aeronautics Systems Analysis Branch, Mail Stop 442.

${ }^{4}$ Systems Analyst, Vehicle Analysis Branch, Mail Stop 451, Senior Member of AIAA.

${ }^{5}$ Aerospace Engineer, Vehicle Analysis Branch, Mail Stop 451.

${ }^{6}$ Aerospace Engineer, Aeronautics Systems Analysis Branch, Mail Stop 442.

${ }^{7}$ Aerospace Engineer, Aeronautics Systems Analysis Branch, Mail Stop 442.
} 
capabilities. This paper is one of a set of papers, developed for presentation within a single conference session, that are intended to highlight the SA capabilities of NASA LaRC Systems Analysis and Concepts Directorate (SACD). This paper discusses methods and tools in the specific areas of uncertainty/risk analysis, quantification, propagation, decomposition, and management, robust/reliability design methods, and extensions of these capabilities into decision analysis methods that support the goals and requirements of NPRs $7120.5^{1}, 8000.4^{2}$ and $8705.5^{3}$. For convenience, this group of disciplines will simply be referred to collectively herein as Decision Support (DS) methods and tools. These DS methods and tools both overlap with, and are distinct from, conventional SA technical processes (described subsequently) and fill critical roles in the SA process.

A companion paper ${ }^{4}$ defines SA as the "unique combination of discipline[s] and skills to work in concert with NASA Headquarters and the NASA Centers to perform studies for decision makers...to enable informed programmatic and technical decisions". The same paper ${ }^{4}$ defines risk analysis as "the process of quantifying both the likelihood of occurrence and consequences of potential future event." Figure 1, taken from Ref. 4, illustrates the NASA LaRC Systems Engineering (SE) and Analysis process.

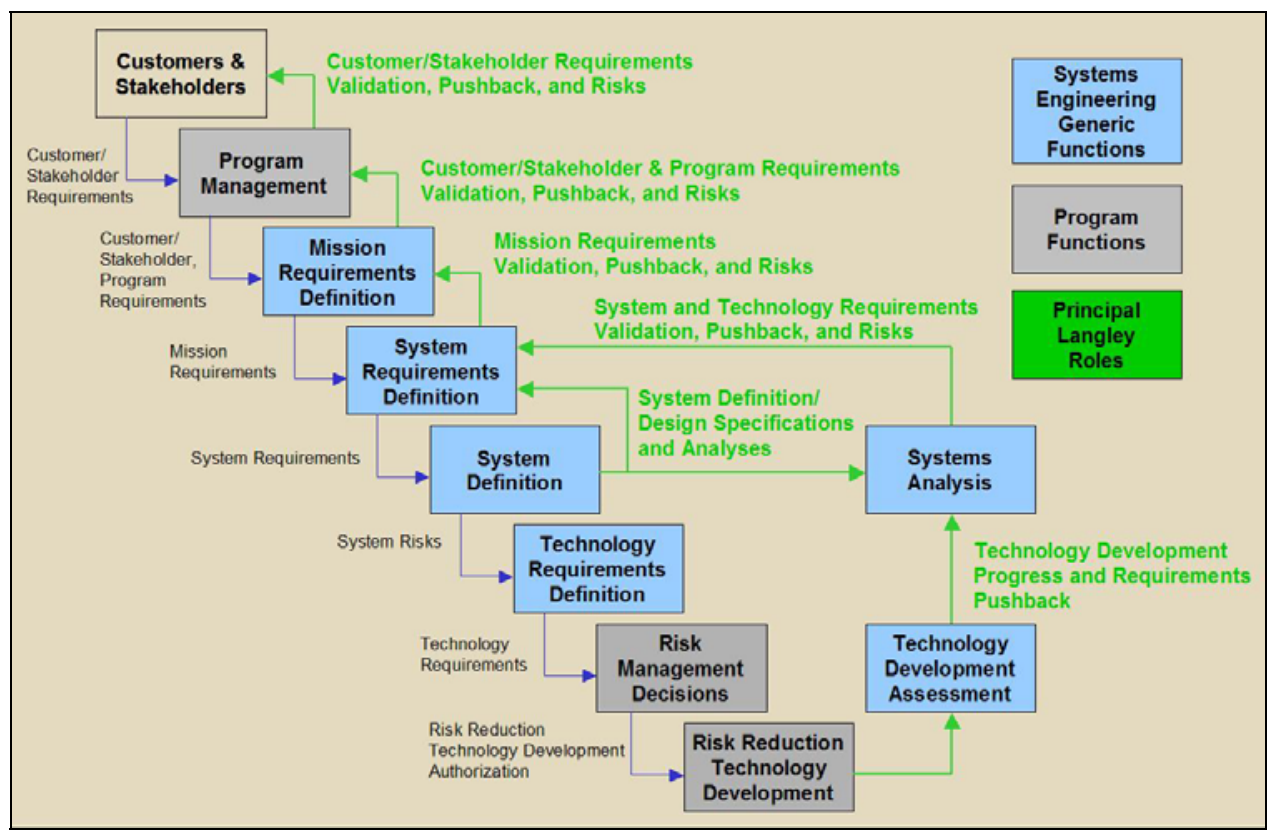

Figure 1. Langley's Systems Engineering and Analysis Process

NASA Procedural Requirements, NPR 7123.1, Systems Engineering Procedural Requirements ${ }^{5}$, defines systems engineering as "a logical systems approach performed by multidisciplinary teams to engineer and integrate NASA's systems to ensure NASA products meet customers needs." The same document also defines systems approach as the application of a systematic, disciplined engineering approach that is quantifiable, recursive, iterative, and repeatable for the development, operation, and maintenance of systems integrated into a whole throughout the life cycle of a project or program." This systems approach includes 17 common technical processes, as shown in NPR $7123.1^{5}$, Fig. 3-1 and 3-2, and as follows:

\section{A. System Design Processes}

1. Stakeholder Expectations Definition

2. Technical Requirements Definition

3. Logical Decomposition

4. Physical Solution

B. Product Realization Processes

5. Product Implementation

6. Product Integration

7. Product Verification

8. Product Validation

9. Product Transition 
C. Technical Management Processes

10. Technical Planning

11. Requirements Management

12. Interface Management

13. Technical Risk Management

14. Configuration Management

15. Technical Data Management

16. Technical Assessment

17. Decision Analysis

The 17 common technical processes are not intended to be performed strictly sequentially. In fact, it is expected that, at least, the Technical Management Processes (items C) take place concurrently with the System Design Processes (items A) and/or the Product Realization Processes (items B). But any of the processes may take place in some combination of sequential and concurrent steps, or all may take place concurrently, as appropriate. Furthermore, it is expected that numerous passes through each of the 17 processes may occur, with iteration cycles and re-entry points established as appropriate. The reader is referred to NPR $7123.1^{5}$ for detailed discussions of each of these common technical processes.

However, simply utilizing a good SA or SE process, such as that described in NPR $7123.1^{5}$ does not ensure that all the customer's requirements can be satisfied within cost, schedule, or safety constraints, or with the tools and methods available. Likewise, satisfying the customer's requirements does not mean that the results were obtained by a systematic, disciplined engineering approach that is quantifiable, recursive, iterative, and repeatable (as defined in NPR 7123.1 ${ }^{5}$ ) for the development, operation, maintenance, and disposal of systems. The two aspects of this problem, customer satisfaction and good SA/SE process, are really mutually independent, though correlated, as shown in Fig. 2, which is fashioned after a typical risk assessment matrix, discussed later in this paper. That is to say, an SA or SE process could produce outcomes in any cell of the matrix in Fig. 2, including four corners of the matrix, of which the lower left corner is the best outcome possible. However, a good SA or SE process should include a negotiation between the developer/provider and the customer, early in project lifetime and throughout, to ensure that a reasonable chance exists to satisfy the customer's requirements within cost, schedule, and safety constraints. The customer should be suspect of any results obtained by a poor and/or undocumented process.

\begin{tabular}{|c|c|c|c|c|c|c|}
\hline & $\begin{array}{l}\text { customer requirement } \\
\text { satisfaction is certified }\end{array}$ & $\begin{array}{c}\text { customer } \\
\text { requirements } \\
\text { satisfied; traceability } \\
\text { is reported }\end{array}$ & $\begin{array}{l}\text { customer } \\
\text { requirements } \\
\text { fully satisfied }\end{array}$ & $\begin{array}{c}\text { customer } \\
\text { requirements } \\
\text { partially satisifed }\end{array}$ & $\begin{array}{l}\text { no attempt to } \\
\text { identify or satisfy } \\
\text { customer } \\
\text { requirements }\end{array}$ & \multirow{6}{*}{ 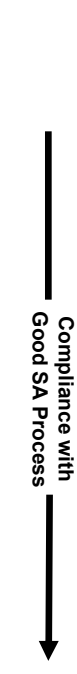 } \\
\hline $\begin{array}{l}\text { poor or nonexistant } \\
\text { SA process }\end{array}$ & $\begin{array}{l}\text { customer happy } \\
\text { process risk } \\
\text { is unknown }\end{array}$ & & & & $\begin{array}{l}\text { customer unhappy } \\
\text { process risk } \\
\text { unkonwn }\end{array}$ & \\
\hline \multicolumn{6}{|l|}{$\begin{array}{l}\text { good SA process } \\
\text { identified and } \\
\text { partially utilized }\end{array}$} & \\
\hline \multicolumn{6}{|l|}{$\begin{array}{l}\text { good SA process } \\
\text { identified and } \\
\text { fully utilized }\end{array}$} & \\
\hline $\begin{array}{l}\text { good SA process } \\
\text { utilization and } \\
\text { process compliance } \\
\text { is reported } \\
\end{array}$ & & & $\begin{array}{l}\text { Process } \\
\text { k Reduction }\end{array}$ & & & \\
\hline $\begin{array}{l}\text { good SA process } \\
\text { utilization is } \\
\text { certified }\end{array}$ & $\begin{array}{l}\text { customer happy } \\
\text { process risk } \\
\text { is minimized }\end{array}$ & & & & $\begin{array}{l}\text { customer unhappy } \\
\text { process risk } \\
\text { is minimized }\end{array}$ & \\
\hline
\end{tabular}

Figure 2. SA/SE Process Risk Assessment Chart

NASA Procedural Requirements, NPR 8705.5, Probabilistic Risk Assessment (PRA) Procedures for NASA Programs and Projects ${ }^{3}$, states that it "is NASA policy to implement structured risk management (RM) processes 
and use qualitative and quantitative risk assessment techniques to support optimal decisions regarding safety and the likelihood of mission success." The same NPR states "PRA provides a framework to quantify uncertainties in events that are important to system safety. By requiring the quantification of uncertainty, PRA informs the decisionmakers of the sources of uncertainty and provides information that helps determine the worth of investing resources to reduce uncertainty." Furthermore, the same NPR also states "PRA results are directly applicable to resource allocation and other kinds of RM decision-making based on its broader consequence metrics." It should be clear that decisions about resource allocation are implied in Fig. 2, because movement from the upper right-hand corner toward the lower left-hand corner of this figure can only be accomplished by the application of additional resources above and beyond those of the current situation. However, NPR $8705.5^{3}$ also states "it addresses technical and safety risk and does not address programmatic risk involving consideration of cost and schedule." Hence, PRA methods and tools are often distinct from the DS methods and tools, focusing on Safety and Mission Assurance. An accompanying paper $^{6}$ discusses analysis techniques other than the PRA. Program/Project Risk Management is addressed in NPR 7120.5 and NPR 8000.4. In project management, the additional resources required for implementing risk mitigations are ideally identified early and funded out of project resource reserves.

The DS methods and tools fill three critical roles in the SA process: 1) provide the evaluations of uncertainty, risk, and decision-making metrics within the analysis or design process, possibly at all phases of the 17 common technical processes above, 2) provide feedback to the system analysis and design processes, and 3) provide feedback to decision makers or stakeholders managing the analysis or design process so that they can make informed choices. The feedback to the systems analysis or design process may take the forms of adjustments or corrections to the constraint and objective metrics within the analysis or design, side constraints on design variables, modifications to the analysis/design process, or even path selection within the system analysis or design process. The feedback to decision makers or stakeholders may take the forms of uncertainty bounds on deterministic results, probabilities or probability distributions, or quantified information about risks. The DS methods and tools can be thought of as an overlay to Fig. 1, which attempts to quantify uncertainty and risk at each step in that process. DS is a quantifying process relied heavily upon in the Risk Analysis step of Continuous Risk Management. Where applicable, commercially available, or government developed software tools are also discussed in this paper.

Recent experience has emphasized the need for NASA to account properly for the uncertainties that are inherent in engineering analyses. For example, during a recent Aeronautics Enterprise Systems Analysis Methods Roadmapping Workshop, it was claimed that "by 2025 we need a design and analysis process with known uncertainty in the analysis covering the full vehicle life cycle for all aeronautic vehicles (subsonic to hypersonic). The process should be variable-fidelity, flexible (customizable, modular), robust (design to confidence level, reliable) and validated." Moreover, the following quotes from the Columbia Accident Investigation Board (CAIB) report ${ }^{7}$, clearly illustrate this need:

The assumptions and uncertainty embedded in this [debris transport] analysis were never fully presented to the Mission Evaluation Room or the Mission Management Team.

Engineering solutions presented to management should have included a quantifiable range of uncertainty and risk analysis. Those types of tools were readily available, routinely used, and would have helped management understand the risk involved in the decision. Management, in turn, should have demanded such information. The very absence of a clear and open discussion of uncertainties and assumptions in the analysis presented should have caused management to probe further.

Likewise, these quotes from the Final Report of the Return to Flight Task Group ${ }^{8}$, Annex A.2, "Observations by Dr. Dan L. Crippen, Dr. Charles C. Daniel, Dr. Amy K. Donahue, Col. Susan J. Helms, Ms. Susan Morrisey Livingstone, Dr. Rosemary O’Leary, and Mr. William Wegner” further amplify this point:

In the case of debris analysis, models for: 1) debris liberation; 2) aerodynamic characteristics of the debris; 3) transport analysis of debris; 4) impact tolerance of the thermal protection system; and, 5) the resultant thermal and structural models of the effects of damage, are all necessary to assess risk. The uncertainties in one model (or system) inherently feeds into and compounds the uncertainty in the second model (or system), and so on. It appears, however, that NASA largely designed these five classes of models without the attention to the interdependencies between the models necessary for a complete understanding of the end-to-end result. Understanding the characteristics of, and validating and verifying, one type of model without examining the implications for the end-to-end result is not sufficient.

Further compounding the modeling challenge is the fact that the models most often used for debris assessment are deterministic, yielding point estimates, without incorporating any measure of uncertainty in the result. Methods exist to add probabilistic qualities to the deterministic results, but they require knowledge of the statistical distribution of the 
many variables affecting the outcome... The probabilistic analysis...is very dependent on the quality of the assumptions made by the developers. Although they evaluated some of the assumptions used by the model developers, the...end-toend "peer review" primarily analyzed whether the output of one model could be incorporated into the next, not the joint probability associated with any given output... without which it is difficult to know the reliability of the result.

Probability distributions are analytic methods necessary when assessing risk. Without an understanding of the likelihood of an outcome, risk acceptance is a judgment based on instinct and experience. But, as the Columbia accident showed, in a high risk environment that involves many unknowns like human space flight, experience and instinct are poor substitutes for careful analysis of uncertainty.

\section{Uncertainty Analysis / Management}

Uncertainty analysis includes the steps of uncertainty quantification, propagation, decomposition, and management, as well as robust design methods and reliability methods. Each of these topics is discussed within the following subsections. Numerous aerospace examples of uncertainty analysis / management from SACD and prior, similar organizations are available and discussed within the following sections.

\section{A. Uncertainty Quantification}

Uncertainty quantification ${ }^{9-14}$ (UQ) can be used to both improve and clarify the evaluations of the probability of an occurrence, and of the consequence of an occurrence within a risk assessment. Uncertainty quantification begins in steps 1 and 2 of the 17 common technical processes above, with the examination of requirements for a given problem to identify "fuzzy" statements, those that cannot be definitively fulfilled. If the requirements include fuzzy statements, the provider should attempt to work with the customer to better define the requirements, or at least consider how one will establish whether the requirement is met or not. One should also examine specific numerical values given within requirement statements to determine if these numerical boundaries represent laws of physics, which cannot be changed, feasible numerical restrictions, or if they are merely desirable limits. If the requirements include desirable limits, the provider should attempt to determine how much the customer is willing to pay to achieve the desired limit, versus something in the neighborhood of the desired limit. For example, it may be the desired limit is only obtainable at very high cost, whereas values in the vicinity of the desired limit may be achieved at a substantially lower cost. In all cases, the requirements should be examined to determine if they unambiguous, make sense, are achievable, and are not conflicting with other requirements.

One should also examine the conceptual, mathematical, algorithmic, and computational models (steps 3 through 5 of the above 17 common technical processes) for physics or behavioral simplifications, temporal and spatial approximations, conversions from continuous to discrete analysis, geometric / spatial / temporal resolution, physics fidelity, and coding errors which can all introduce uncertainties and/or errors into the process. The executable models and data sets that will be used in evaluating the performance, cost, schedule, and safety metrics of interest for the problem should be examined for incorrect input data, incorrect usage, and incorrect interpretation of results (steps 5 through 9 of the above 17 common technical processes). These examinations should attempt to identify inputs to, and parts of, the process that are unknown, variable, or outside of the control of the analyst or designer. Having identified these which items contribute uncertainty to the process, one should then attempt to quantify the amount of uncertainty associated with each source of uncertainty. This might be as simple as saying there is possibly a $10 \%$ error in this number, or that a certain input potentially has a lowest possible value, a highest possible value, and a most likely value. In general, the more information that can be used to describe the range of the input and model uncertainties, and the distributions associated with the input variables or model approximations, the better. These input and model uncertainties can then be propagated through the analysis and design tools and methods to produce distributions of the output metrics of interest. The uncertainty in the outputs can then be decomposed into the most significant and least significant sources, and finally managed. It is important to note, however, that the entire uncertainty evaluation, propagation, decomposition, and management process is limited by the impact(s) of the most uncertain item(s) within the process.

Uncertainty quantification is generally a statistical analysis of available input data, related to a particular source of uncertainty, for an analysis or design process. The goal of this activity is to determine the type of probability distribution to use, and the values of parameters which can be used to define the probability distribution or probability density function (PDF), and its accompanying cumulative distribution function (CDF), the integral of the PDF. The simplest forms of uncertainty quantification would rely on knowing only a mean value and a standard deviation for which one might assume a normal distribution, or knowing only the minimum and maximum values, one might assume a uniform distribution over the data range. But the choice of a PDF type should not be arbitrarily made, because this choice can have a significant affect on the outcome distribution. Various commercially 
available tools can be used in this activity, such as Model Center from Phoenix Integration (http://www.phoenixint.com/), the Microsoft Excel add-in called Crystal Ball from Decisioneering, Inc. (http://www.decisioneering.com/index.html), the Microsoft Excel add-in called @Risk from Palisade Corporation (http://www.palisade.com/Default.asp) or the PredictionProbe, Inc. tool called Distribution Probe (http://www.predictionprobe.com/index.htm), and many others. These tools generally accept sets of input data, allow the user to fit various PDF types to the input data, determine the optimal parameters within a PDF type to fit the input data, and rank the resulting distribution fits to allow the user to choose the best PDF to model their data. Another commercially available tool called Design Expert from Stat-Ease ${ }^{15-16}$, Inc. will perform normality checks on the data set to determine if the data can be well modeled with a normal distribution. Ideally, having identified and selected a best fit distribution, and perhaps several other possible distribution fits, this set of uncertainty distributions should each be propagated through the analysis or design process to determine if the output distribution is sensitive to the choice of the input distribution. If great variability is observed in the output distribution as the input distribution changes, further validation of the input distribution should be performed, or more input data samples obtained, in order to ensure that the input data distribution has been correctly modeled.

One recent uncertainty quantification effort within SACD involved the use of the Crystal Ball software to provide a probabilistic characterization of the uncertainty in launch insertion accuracy and launch date delays. Periapse, apoapse, and inclination accuracy data was analyzed from approximately 110 combined launches of Pegasus XL, Atlas II, and Delta II launch vehicles. Launch delay data for small satellites, other missions, and the combined set of small satellites and other missions were also analyzed. Each analysis resulted in a "best fit" PDF, by one of several choices of methods. The resulting PDF were then used as inputs to a probabilistic satellite orbital lifetime analysis ${ }^{17}$.

\section{Jet Flap Airfoil Uncertainty Analysis}

Another ongoing uncertainty quantification effort within SACD involves the application of a post-processor Analysis of Variance (ANOVA) technique ${ }^{18-20}$ within the Design-Expert software (version 6.0.10) from Stat-Ease, Inc. ${ }^{15-16}$ to characterize the aerodynamic performance uncertainty for a jet flap wing configuration proposed for advanced military aircraft. The jet flap wing concept would use trailing edge blowing to induce circulation around the wing, changing its lift, drag, and pitching moment characteristics ${ }^{21}$. The ANOVA study was conducted to quantify the uncertainty of the computational results relative to the experimental results. Analysis of variance is a statistical technique that subdivides the total variation of a set of data into component parts associated with specific sources of variation. This study proved to be a fairly benign test of the ANOVA capabilities.

To perform the ANOVA, factors such as the Mach number, blowing coefficient, angle of attack, and a grid density metric, which potentially affect the computed lift and pitching moment coefficient, compared to the measured data, were identified and input to the software. Ranges of interest for the factors were input to the software along with the discrete computed or measured values of the factors. Also input were the computed or measured lift and pitching moment coefficient associated with the input values of the factors. In a matter of seconds, the Design-Expert software calculated a numerical model of user-specified order (linear, 2-factor interference, quadratic, or cubic) to fit the input data, residuals (differences) between the model and actual data were produced at each of the input conditions, and uncertainties (in the form of Least Significant Differences or LSD) for experimental, computational, and combined experimental / computational data sets were computed. The LSD computed by the software indicate the smallest resolvable differences in the functional values (lift and pitching moment coefficient) given just the input data points from selected data sets. The software also provides a collection of diagnostics which evaluate the suitability of the input data set for use within the ANOVA process and which examine the behavior of the resultant data, suggesting transformations which should be applied to the data to reduce the LSD.

Figures 3-6 illustrate some of the key features and results from the uncertainty analysis studies. Figure 3 illustrates the uncertainty analysis for the computational grid sensitivity study, described in Ref. 21 at Mach=0.3. The curve in the plot represents the approximate quadratic numerical model, which the Design-Expert software fitted to the input data. Design points are shown where input data were provided. As in Ref. 21, Fig. 6, the lift coefficient is shown as a function of the grid density metric, but here with $95 \%$ confidence least significant difference (LSD) error bars added. The blue square simply indicates where on the curve the LSD is applicable. The LSD bars indicate the smallest resolvable differences in the functional values (lift coefficient) attributable to changes in the grid density metric, given just the selected input data points. In this case, the LSD is about 0.0050 , which is 


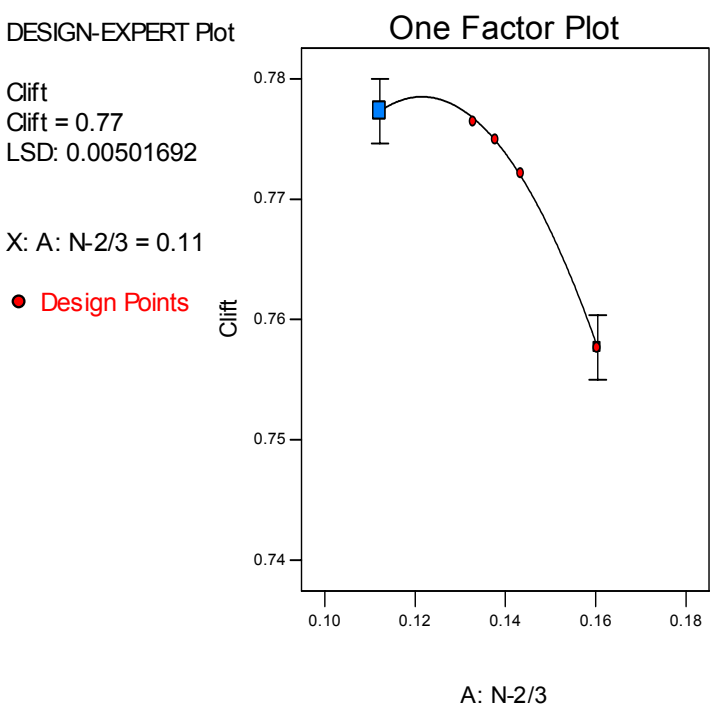

Figure 3. Uncertainty Analysis, Grid Sensitivity Study at Mach $=0.3$, Alpha $=6.0^{\circ}$.

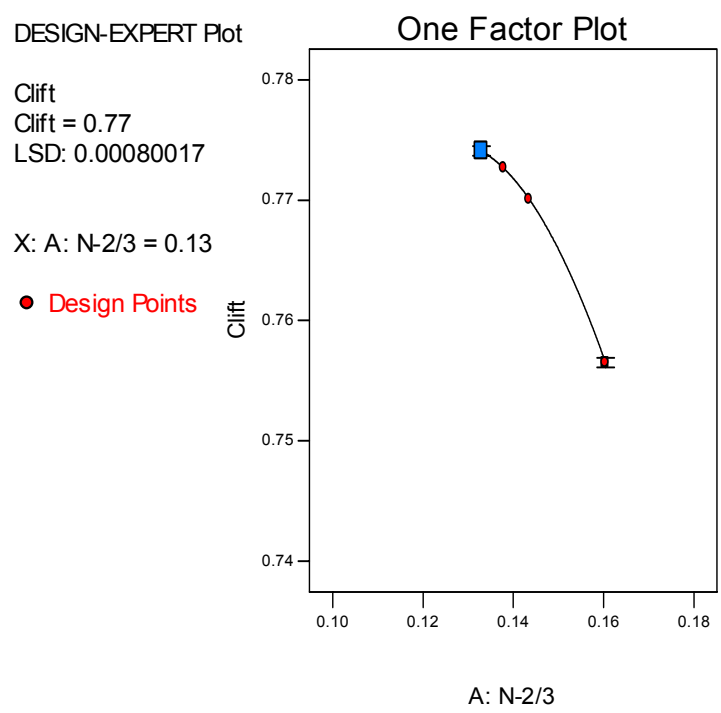

Figure 4. Uncertainty Analysis, Grid Sensitivity Study at Mach $=0.3$, Alpha $=6.0^{\circ}$, finest grid data excluded.

divided by two and added or subtracted from the calculated numerical model value to obtain high and low $95 \%$ confidence bounds. This means the potential lift error due to grid density is about $+/-0.0025$. On the finest grid, the actual lift coefficient at this grid density (0.7750) is expected to be between 0.7724 and 0.7775 with $95 \%$ confidence; there is a $5 \%$ chance that the actual lift on the selected grid could be outside of these bounds. The actual lift coefficient, in this case, is well within the $95 \%$ confidence bounds. The actual lift coefficient value on the selected grid (0.7741, second from left) also lies well within the $95 \%$ confidence interval for that grid, where the approximate model value is 0.7744 , and the range is between 0.7719 and 0.7769 with $95 \%$ confidence. However, in this example, because of the clearly parabolic shape of the approximate model curve shown in Fig. 3, and the fact that several input values of lift coefficient are between 0.7700 and 0.7800 , the confidence interval is broader than might be expected and is also broader than desired. As shown in Fig. 4, the uncertainty can be reduced on the selected grid, indicated by the blue square, simply by ignoring the finest grid results; this removes ambiguity about the behavior of the lift coefficient as the grid density is increased (moving toward left on the plot), and allows for a prediction of the actual lift coefficient on the selected grid to be within $+/-0.0004$ of the approximate model value 0.7741 , or that the actual lift coefficient is expected to be between 0.7737 and 0.7745 , with $95 \%$ confidence. This is a reduction of the prediction uncertainty by more than a factor of ten, by simply removing some ambiguity in the data presented to the software. Similar results for the grid sensitivity study at Mach $=0.8$ (not shown), again excluding the data from the finest grid, resulted in a 95\% confidence interval (LSD) of about 0.0070 .

Figure 5 illustrates a different way to perform the grid sensitivity uncertainty analysis. In this example, both Mach=0.3 and Mach=0.8 have been considered together, with data from the finest grid again excluded. The lift coefficient was described to the Design-Expert software as a function of the numerical (continuous) grid density factor and a categorical factor of Mach number, which could only take on the discrete values of 0.3 or 0.8 . In this case, since the lift behavior with grid density for both grid studies has the same functional form, the combination of the data allows the software to better resolve the potential error due to grid density. The maximum LSD of the combined data set is now about 0.0018 , meaning the potential lift coefficient error is only about $+/-0.0009$ due to grid density.

Figure 6 illustrates the uncertainty analyses for two similar, but different, input data sets. Both computational and experimental Mach= 0.3 data with blowing coefficient up to about 0.07 are analyzed as a function of the blowing coefficient. The difference between computational and experimental data is treated again as a categorical factor, which can only take on two distinct values (i.e., Type $=$ Computational or Experimental), whereas the blowing coefficient is treated as a numerical (continuous) factor. In this example, repeated experimental data was included, only incremental jet effects (Jet On - Jet Off) are considered, and a power transformation applied to all the input lift coefficient data $(0.00684186$ was added to each input lift coefficient value and the sum, for each input data point, is 
then raised to the 1.63 power), as suggested by the software. This analysis parallels the lift augmentation behavior as a function of the blowing coefficient, reported in Ref. 21, Fig. 18, and now analyzed in this transformed space. The LSD is about 0.0113 in the transformed space, which can be shown to reduce the LSD to about 0.0067 in the untransformed space. The same data (repeated experimental points, and incremental jet effects) analyzed in the untransformed space (not shown) yielded an LSD of about 0.0838 - a significantly larger uncertainty band.

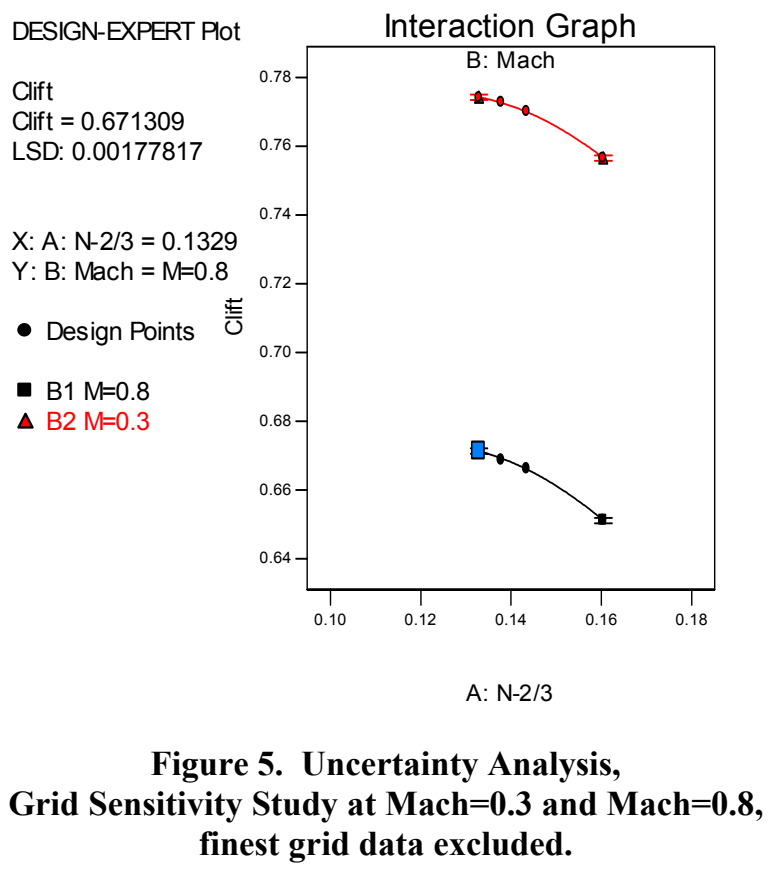

\section{Hurricane Track Prediction Uncertainty Analysis}

The ANOVA technique ${ }^{15-16,18-20}$ was also applied to two much more challenging data sets. In the first example, ANOVA was applied to historical hurricane track data. This was presumed to be a very challenging test of the ANOVA method and was conducted to determine if the technique could compete with, or improve upon, current hurricane track forecasting methods. Hurricane track and intensity data, dating from 1851 through the beginning of 2006, was obtained from the U. S. Department of Commerce, National Oceanic and Atmospheric Administration (NOAA), National Hurricane Center (NHC), website (http://hurricane.csc.noaa.gov/hurricanes/download.ht $\mathrm{ml}$ ) and loaded into Microsoft Excel for preprocessing. The pre-processing step consisted of simply rearranging the data in proper format for use with the Design-Expert software, and a few minor calculations, such as creating a time stamp for from the year, month, and date entry of each record. A total of 32756 recorded measurements, a subset of the data back to only 1885 (the complete data set was too large for current version of the software), taken at six-hour intervals during the lifetimes of 1119 North Atlantic

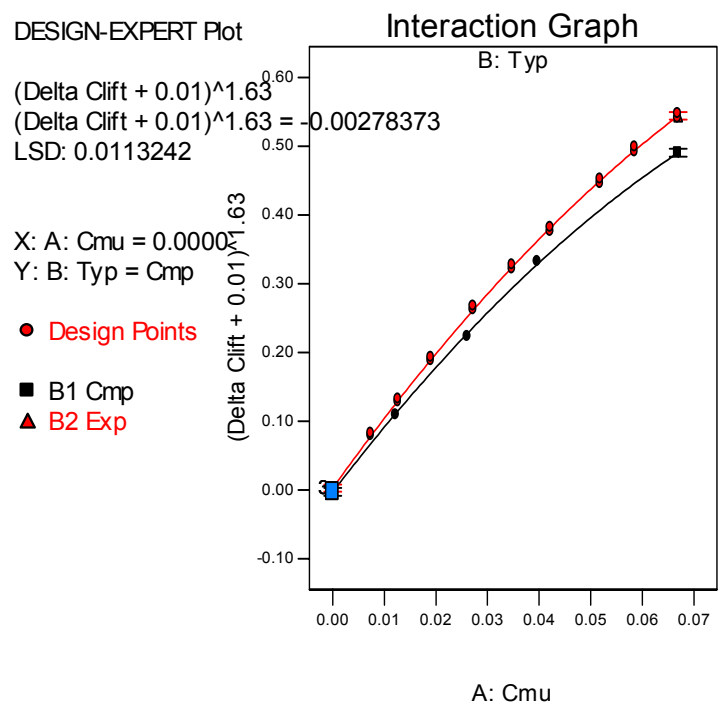

Figure 6. Uncertainty Analysis, Blowing Coefficient Sensitivity Study at $\mathrm{Mach}=0.3$, Alpha $=6.0^{\circ}$, repeated experimental data included, power transformation applied, $\mathrm{k}=\mathbf{0 . 0 0 6 8 4 1 8 6 ,} \lambda=1.63$.

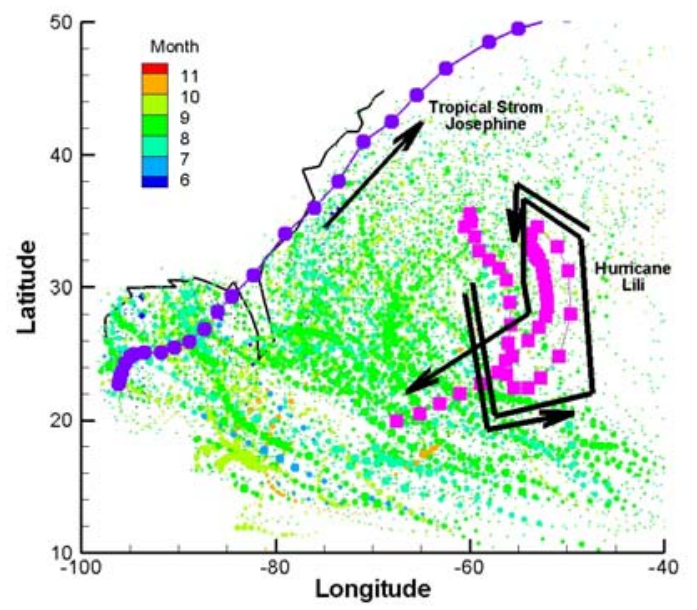

Figure 7. North Atlantic Basin Hurricanes, Sized by the Saffir-Simpson Hurricane Scale. 
Basin hurricanes, tropical storms, and tropical depressions, were provided to the Design-Expert software for analysis in this demonstration. Sample hurricane track data only (as discrete points) are shown in Fig. 7, along with a crude approximation to the eastern seaboard of the United States of America. The point coloring in Fig. 7 indicates the month of the year, and the point size is correlated with the Saffir-Simpson scale (http://en.wikipedia.org/wiki/SaffirSimpson_Hurricane_Scale).

The storms analyzed were not chosen in any particular way and thus included a wide variety of storm tracks. For example, data analyzed include the path of Tropical Storm Josephine (Oct. 1996) and that of Hurricane Lili (Dec. 1984), as shown in Figure 7. The path of Tropical Storm Josephine is highlighted in purple with large connected circular symbols; the storm formed near the western edge of the Gulf of Mexico and moved steadily to the North East, and happened to pass very close to Hampton, VA. This storm had the western-most starting point of all the storms analyzed. The path of Hurricane Lili is highlighted in Fig. 7 in pink with large connected square symbols; the storm formed in the mid North Atlantic, moved to the South and East, circled back to the North, then finally moved to the South and West.

Several factors were chosen from data available that could potentially influence the track and intensity of hurricanes as they develop and progress. The factors chosen for this demonstration were: 1) a year fraction computed from the month and day, divided by the maximum number of days in the year of record - provides a timeline through a typical year to track cyclic behavior on a seasonal basis, 2) the duration of the storm as measured in six-hour increments, 3) the starting date including the year, month, and day of the month - provides a timeline through all the records to track cyclic behavior on an annual basis, 4) the starting longitude value, 5) the starting latitude value, 6) the starting maximum wind speed, 7) the current longitude value, and 8) the current latitude value. The responses for this demonstration were the actual hurricane longitude and latitude values 24 and 48 hours after the input record. No information was available within this data set regarding local atmospheric or water temperatures, or short duration weather patterns which would logically be expected to influence the storm development and path. The goal of this exercise was to determine if the software could be used to discern correlations and interactions among the factors and seemingly chaotic response data to make an accurate prediction of the storm location 24 to 48 hours in advance. The stated goal of the NOAA/NHC is an average $(50 \%$ confidence interval) 48-hour track forecast prediction error of no more than 125 nautical miles (nm), to be achieved by 2009. Their achieved average 48-hour track forecast prediction error was just $107 \mathrm{~nm}$ in 2003.

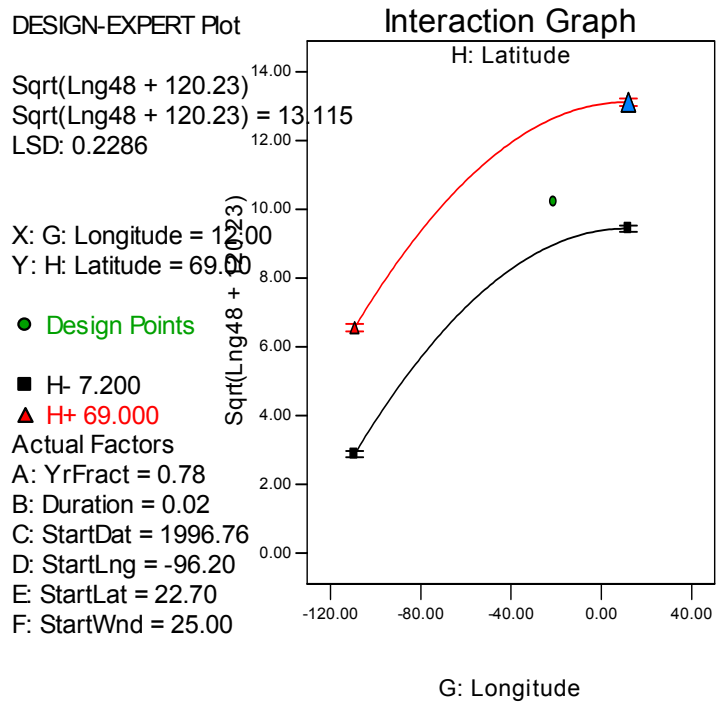

Figure 8. Design-Expert Plot of 48-Hour Prediction of Storm Longitude in Transformed Space.
Figure 8 illustrates the interactions of the current longitude and latitude (as two of the eight interacting factors within the model) on the 48-hour prediction of longitude and latitude, within a transformed space suggested by the software (a square root transformation with offset of 120.23). The curves are the approximate model generated by the software by fitting a modified quadratic to the input data at each of the extreme input values of current latitude, for given values of current longitude (across x-axis), with the other six factors fixed at the values shown in the plot. That is to say, for a given current longitude between $12^{\circ}$ and $-109.3^{\circ}$, one can interpolate the least significant difference (LSD) for the 48-hour predicted longitude between the two curves for a given current latitude between $7.2^{\circ}$ and $69^{\circ}$, and with the other six factors fixed at the values shown. The circle represents an actual input data point, in this case, the last point considered in the path of Tropical Storm Josephine (current longitude $=-15.5^{\circ}$ and current latitude $=55.5^{\circ}$ ). The error bars are the LSD measure, defined previously. In this case, the maximum LSD for the conditions of interest is 0.220864 in the transformed space, which can be shown to translate into a $95 \%$ confidence interval about 392.1 miles $(340.7 \mathrm{~nm})$. That is to say, given the data analyzed, there is only a $5 \%$ chance that the actual 48 -hour longitude predicted location would be outside of these bounds. A similar point ( $95 \%$ confidence) from the NOAA/NHC web site (http://www.nhc.noaa.gov/verification/verify2.shtml) yields about a $300 \mathrm{~nm}$ 48-hour forecast error. The Design-Expert / ANOVA track error 50\% confidence interval would be about $117.2 \mathrm{~nm}$, compared to the NOAA/NHC stated goal of $125 \mathrm{~nm}$ from above. Although the Design-Expert / ANOVA error estimate at 95\% is

$$
9
$$

American Institute of Aeronautics and Astronautics 
about $13 \%$ greater than a comparable number from NOAA/NHC, the $50 \%$ confidence intervals are comparable. The Design-Expert / ANOVA estimate was obtained very quickly, with a desktop workstation, and with no attempt to characterize the storm tracks. It is thought that this demonstration represents an encouraging start for further investigation. The 48-hour latitude prediction exhibits definite non-normal behavior that would require further investigation.

\section{Solar Flux Intensity Prediction Uncertainty Analysis}

Another application of the ANOVA technique ${ }^{15-16,18-20}$, still in progress, is to determine if the Design-Expert tool can be used to aid in the long-range (10-50 years) prediction of solar flux intensity ${ }^{17}$. Numerous efforts were under taken using a variety of techniques, as the problem proved to be quite challenging. Measured data was provided from each month for the period starting with January 1953 and ending with August 2005. Measured solar flux intensity data was assumed to be exact, having zero measurement error, since no source to quantify the measurement error was readily identified; this assumption is merely a convenience, not a requirement for the following analysis. In this case, a simple, 3-point average smoothing scheme, given in Eqs. (1),

$$
\begin{gathered}
\operatorname{smooth}(\mathrm{n})=(\operatorname{measured}(\mathrm{n}-1)+\operatorname{measured}(\mathrm{n})+\operatorname{measured}(\mathrm{n}+1)) / 3 \\
\operatorname{smooth}(1)=\operatorname{measured}(1) \\
\operatorname{smooth}(\mathrm{np})=\operatorname{measured}(\mathrm{np})
\end{gathered}
$$

was applied to the measured flux data for all interior points, $\mathrm{n}=1$, $\mathrm{np}-1$, where $\mathrm{np}$ is the number of measured data points (633). The smoothing scheme was applied for 91 iterations, until the gross character of the measured data was captured by the smoothed data, without all the high frequency variation present in the measured data, as shown in Fig. 9.

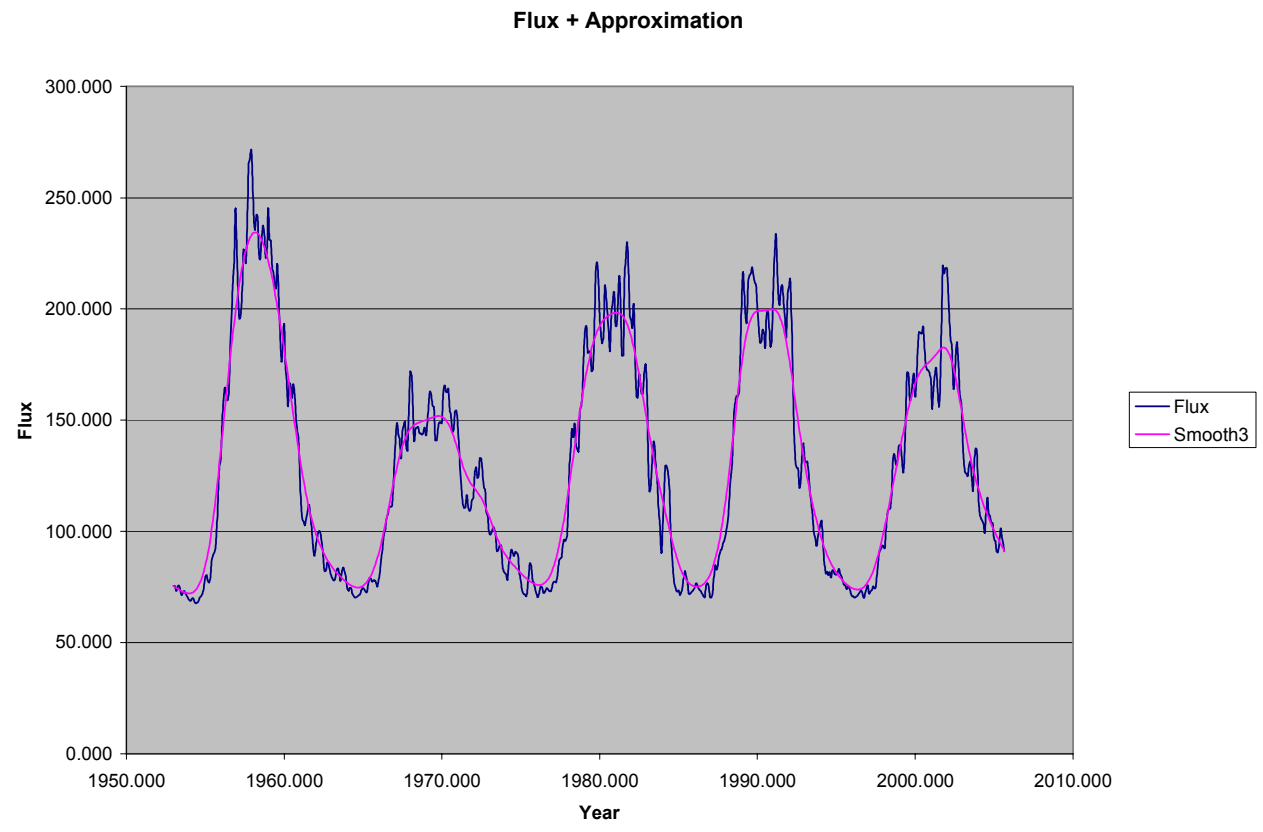

Figure 9. Measured and Smoothed Solar Flux Intensity

The variations of the measured data (Flux) about the smoothed data (Smooth3) in Fig. 9 should naturally take on a normally-distributed character, with a mean value of zero, by virtue of the smoothing process applied. The calculated standard deviation was about 11.42 . The normality assumption can be verified by analyzing the deltas $($ Delta $=$ Flux - Smooth3) as a function of real number Year, which is the sum of an integer measurement year, and a fraction of a measurement year (for months 1-12, divided by 12) within the Design-Expert software, as shown in Fig. 10. In this case, the software suggested that a square root transformation, with a constant of 29.1863 added to each input Delta to ensure a positive-definite response, be applied to the input delta data, which has been done. The approximately linear behavior of the input data over most of its range in Fig. 10 indicates that the data can be 
reasonably assumed to be a normally-distributed about the mean value.

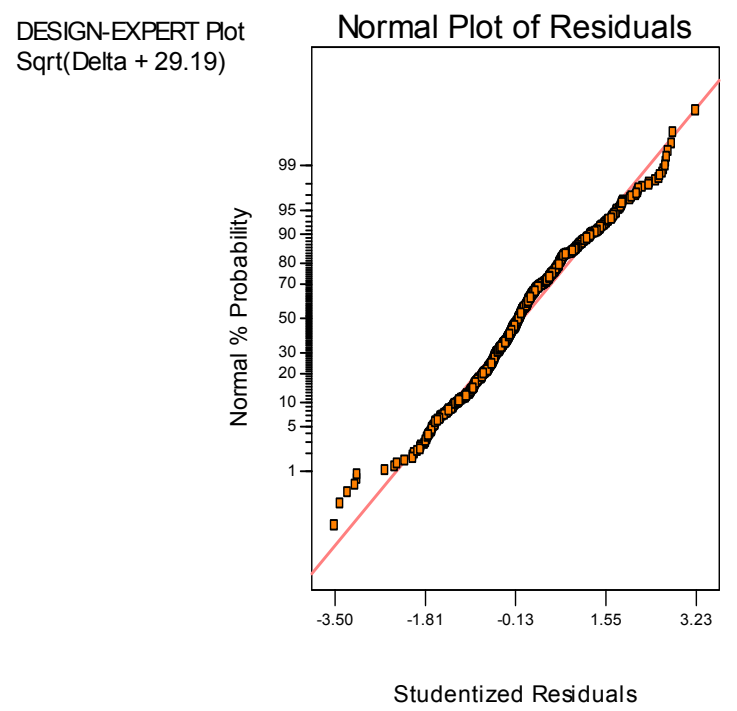

Figure 10. Normality Assessment of Measured Smoothed Solar Flux Intensity Deltas

The smoothed data is still a highly complex function of varying amplitude, with multiple frequency and phasing components present. An attempt to optimally model the smoothed data function with up to ten sine wave components, each having a variable amplitude, frequency, and phase angles (i.e., 30 design variables), proved to be unacceptable. However, by rearranging the smoothed data to be Year as a scatter plot function of Smooth3, as shown in Fig. 11, the periodicity of the certain points within the smoothed data set could then be easily analyzed. A truly periodic function would have a linear increase in the year associated with local minima or maxima, as a function of the cycle being investigated. Having rearranged the data in this fashion, it was a simple matter to determine the Year for the first M points near each local minima or maxima.

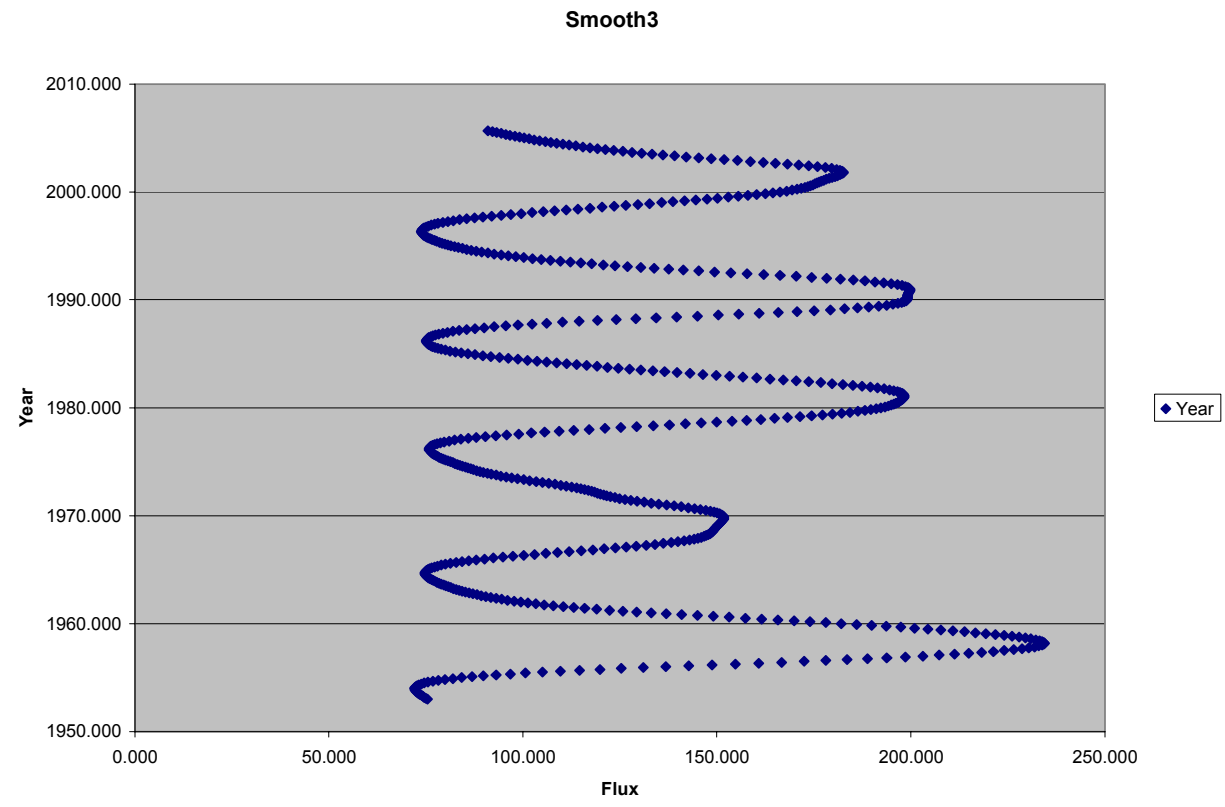

Figure 11. Years Corresponding to Smoothed Solar Flux Intensities

11

American Institute of Aeronautics and Astronautics 
Thus, the Year associated with each of the minimum points for the five local minima in Fig. 11 could be input into the Design-Expert software as a function of the cycle number to determine if the points exhibited a linear behavior. Similarly, the two, three, or eight, Years associated with most-minimum points of the five cycles (or the one, two, three, or eight Years associated with the most-maximum points of the five cycles) could also be analyzed to determine if the linear relationship improved or degraded, with repetitive entries for the extreme points of each cycle. This allows for the possibility that the some measurement error, either in time or intensity, of the extreme points might occur. In fact, the local minima and maxima exhibited highly periodic behavior, which improved with repetitive entries for each extreme point, as shown in Fig. 12. However, the frequency and phasing of the minimum points was slightly different than that of the maximum points. This leads to the shape changes which depart from true sine wave for the smoothed data. Specifically, the cycle minima were well approximated by the Design-Expert software, with eight points provided at each minimum, by the following periodic model

$$
\text { Smooth3-Min }=+1943.56671+10.62499 * \text { Cycle }
$$

Similarly, the cycle maxima were well approximated by the Design-Expert software, with eight points provided at each maximum, by the following model

$$
\text { Smooth3-Max }=+1947.87502+10.81665 * \text { Cycle }
$$

The reader should note that the LSD in the figure is about 0.3457 , which implies that minimum and maximum points are known to within about +/- two months within a 10.6 to 10.8 year cycle. Employing the periodicity implied by the above two relationships, numerous estimates of the Year associated with each minimum and maximum can be computed. The frequency and phase shift effects implied by the above realtionships determine sets of earliest and latest possible start dates of the five cycles of measured data, as shown in Table 1.

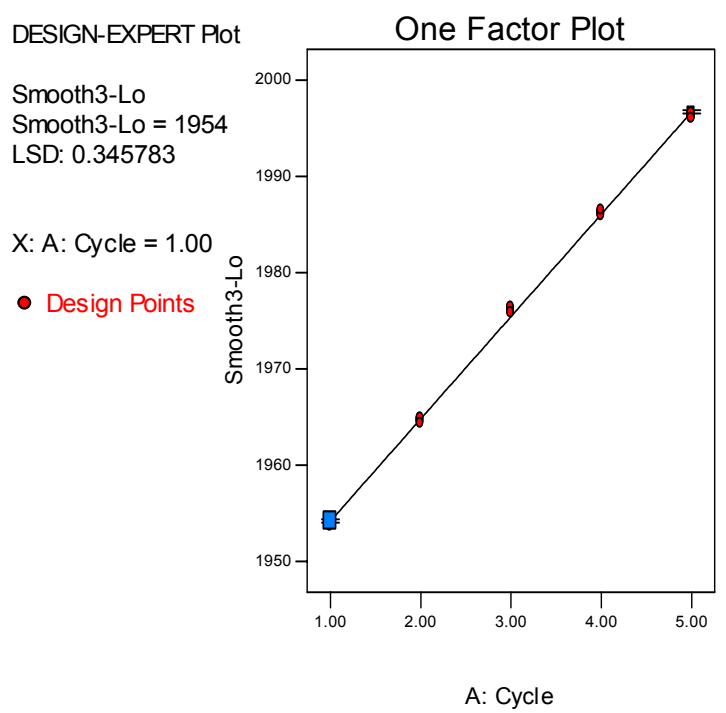

\section{Table 1. Earliest and Latest Solar Flux Intensity Minima and Maxima}

\begin{tabular}{|c|c|c|}
\hline & Earliest & Latest \\
\hline Min1 & 1953.254 & 1954.192 \\
\hline Max1 & 1958.667 & 1959.504 \\
\hline Min2 & 1964.079 & 1964.817 \\
\hline Max2 & 1969.492 & 1970.133 \\
\hline Min3 & 1974.904 & 1975.450 \\
\hline Max3 & 1980.317 & 1980.767 \\
\hline Min4 & 1985.729 & 1986.083 \\
\hline Max4 & 1991.142 & 1991.400 \\
\hline Min5 & 1996.550 & 1996.717 \\
\hline Max5 & 2001.958 & 2002.033 \\
\hline
\end{tabular}

Figure 12. Actual and Approximate Periodicity of Smoothed Solar Flux Intensity Minima

Having determined the earliest and latest Years for which the smoothed minima and maxima data exhibited periodic behavior, the measured flux data for each of the five cycles could then be extracted from the original data set at these points, and overlaid. Then, average cycle data could be computed from the existing five cycles of data, along with deltas of the measured data from the average cycle data. Local standard deviations of the deltas for measured data relative to the average cycle data, as a function of Time through the cycle (ranging from a period of 10.625 to 10.817 years, depending on the Design-Expert model chosen), could then be statistically analyzed with the 
Design-Expert software. The deltas of measured data relative to the average cycle data were found to be normally distributed about the mean values (the average cycle data) to a high degree of confidence. The variations between the measured flux data and average cycle data were almost entirely captured within +2 sigma (local standard deviations) and -1 sigma of the average cycle curve data, as shown in Fig. 13, which is just one sample (but quite representative) of the many possible cycle extractions and overlays.

Overlayed Cycles with Average and +2/-1 Local Sigma Bounds

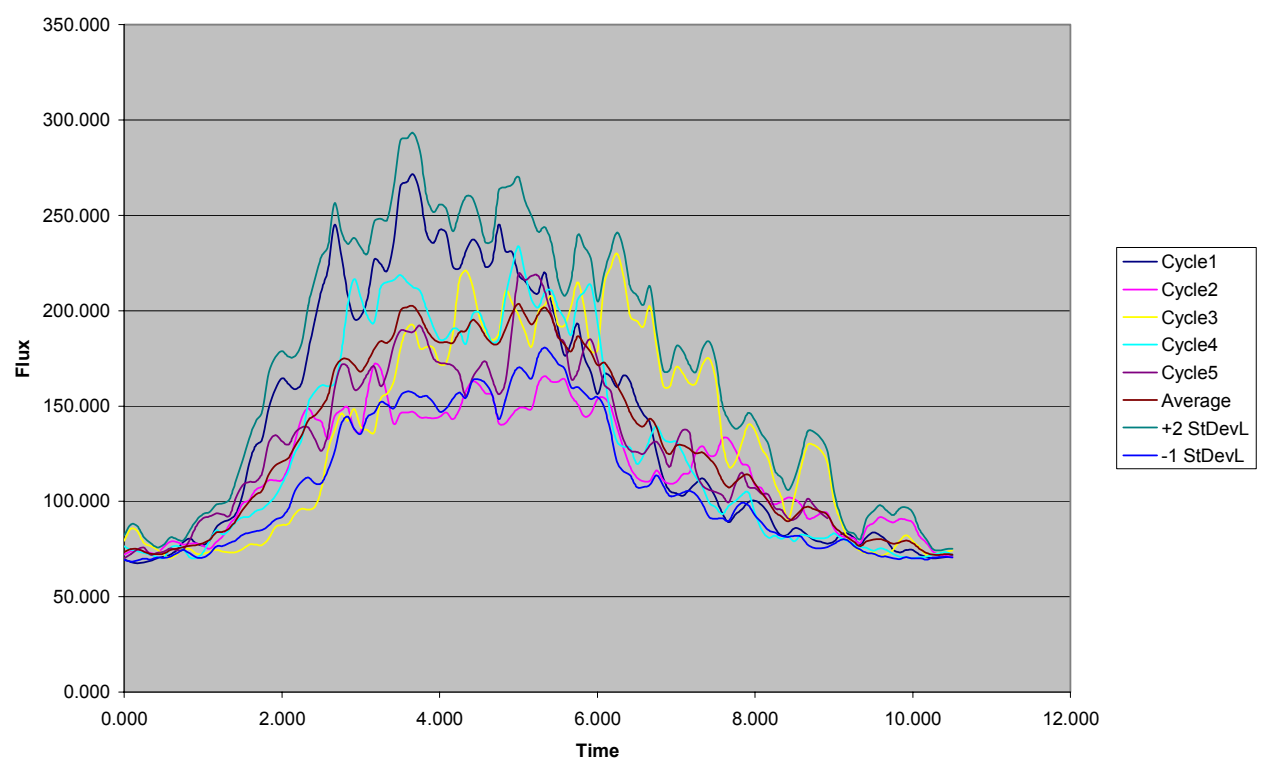

Figure 13. Overlapping Measured and Average Solar Flux Intensity with +2/-1 Local Standard Deviation Bounds

Thus, to a high degree of confidence (about 95\%), predictions about future solar flux intensity measurements can be made by simply adding a normal distribution of delta fluxes with a mean value of zero, and a prescribed standard deviation (StDevL) as a function of time throughout a cycle, to Average cycle flux data determined from $50+$ years of measured data. Although the variation from a possible minimum predicted flux to a possible maximum predicted flux at some future time is quite large, the confidence level associated with this prediction is quite high. A conservative estimate would be to simply to use the average cycle data plus two times $\operatorname{StDevL}(t)$ for a given time in a future cycle as a best guess about the future flux intensity. It is expected that this prediction technique is valid for at least 50 years into the future, at which point the frequency and phase shift effects present in determining the average cycle data and the earliest and latest start dates of cycles may become significant enough to reduce the confidence associated with the prediction.

\section{B. Uncertainty Propagation}

There are numerous methods to perform uncertainty propagation through an analysis or design process. These can be generally classified as First- or Second-Order Reliability Methods (FORM/SORM), simulation methods, importance sampling methods, response surface methods, and mean value based methods, using the language of the PredictionProbe, Inc. tool UNIPASS (http://www.predictionprobe.com/index.htm). The FORM and SORM methods are good when the there is only a very small expected probability of failure, and differ only in whether a linear or quadratic approximation is used to approximate the constraint boundaries at the most probable point(s) (MPP) of failure. Simulation, sampling, and mean value based methods work best when there is a large expected probability of "failure" and with many analysis or design input variables; in this case, the failure mode is generally thought of as degraded performance, rather than a catastrophic failure. Hence, these methods are suitable for robust design applications where a typical goal is to minimize the output sensitivity to variation of the uncertain inputs. Response surface methods are generally used when the objective or constraint functions are very expensive to evaluate, and hence only a limited few "high fidelity" answers representing the real world behavior are available. Several of these classes of examples will be discussed in following sections of this paper. 


\section{Uncertainty Propagation in Stability and Control (S\&C) Derivative Application}

One study of uncertainty propagation involved the application of the automatic differentiation (AD) of FORTRAN (ADIFOR) tool, version 2.0D ${ }^{22-27}$ to the Panel Method Ames Research Center (PMARC) code ${ }^{28}$ version 14.10, a low-order panel method CFD code that was modified by applying AD to enable efficient computation of exact first and second force and moment derivatives with respect to a wide variety of code inputs. ADIFOR 2.0D was applied to PMARC by using a wide variety of input variables as potential independent variables of differentiation. The PMARC body and wind axis forces and moments were used as the dependent variables of differentiation. Since uncertainty estimates of all S\&C parameters (forces and moments and their derivatives) are normally requested by most control law designers, the computation of both first and second derivatives with respect to selected independent variables of differentiation are demonstrated; the latter are used to enable the propagation of known or assumed input variable uncertainties through the code to determine the uncertainty effects on the computed forces and moments. The relationship for uncertainty propagation ${ }^{11-12}$ as a function of normally distributed, random variables is given in Eqs. (2), as follows:

$$
\begin{aligned}
& \sigma_{j}=\sum_{i=1}^{m}\left(\frac{\partial F_{j}}{\partial x_{i}} \sigma_{i}\right), j=1, n \\
& m=\text { number of random inputs } \\
& n=\text { number of output functions } \\
& \sigma_{i}=\text { standard deviation of } \\
& \text { the random inputs }
\end{aligned}
$$

In this case, the output function $F$ may be any of the computed body or wind axis forces and moments, or their first derivatives with respect to parameters such as the angle of attack, angle of sideslip, and the steady rotational rates. As described in Ref. 13, the original and ADIFOR-generated PMARC code versions were executed for an array of oscillatory and rotary aircraft motions to compare with data generated for a $10 \%$ scale F-16XL model tested $^{29}$ in the NASA LaRC 14- by 22-Foot Subsonic Tunnel.

Derivatives of the forces and moments and their first derivatives with respect to angle of attack were differentiated again with respect to angle of attack $(\alpha)$ and a constant pitch rate, $Q$ (or $q)$. Uncertainties with respect to the angle of attack were computed and propagated through the code, proportional to variable gradients with respect to the uncertain variable, via Eqs. 4. The results from this study are shown in Table 2. The table shows the variable name, the uncertain variable name, the computed output variable sigma (from Eq. 1), and one-, two-, and three-sigma (representing 84.1, 97.7, and 99.9\% , respectively) low/high uncertainty bounds for each variable, as well as the mean value for each variable. Table 2 illustrates a typical preliminary uncertainty analysis for the F16XL configuration. The uncertainties in $C_{L}, \frac{d C_{L}}{d \alpha}$, and $\frac{d C_{L}}{d q}$ (with $\alpha$ in degrees and $q$ in degrees per second) are presented as calculated from first and second derivatives of $C_{L}$ with respect to $\alpha$ and $q$, assuming $\sigma_{\text {input }}=0.1$ for both $\alpha$ and $q$. The same capability can be easily applied to all of the forces and moments in both the wind and body axis systems, for any of the independent variables of interest.

Table 2. Sample uncertainty analysis for F-16XL all values of $\sigma_{\text {input }}=0.1$.

\begin{tabular}{|c|c|c|c|c|c|c|c|c|c|}
\hline Variable Name & $\begin{array}{c}\text { Uncertainty } \\
\text { Variable }\end{array}$ & $\begin{array}{c}\text { Computed } \\
\text { sigma }\end{array}$ & $\begin{array}{c}\mathbf{9 9 . 9 \%} \\
\text { Low }\end{array}$ & $\begin{array}{c}\mathbf{9 7 . 7 \%} \\
\text { Low }\end{array}$ & $\begin{array}{c}\mathbf{8 4 . 1 \%} \\
\text { Low }\end{array}$ & $\begin{array}{c}\text { Mean } \\
\text { Value }\end{array}$ & $\begin{array}{c}\mathbf{8 4 . 1 \%} \\
\text { High }\end{array}$ & $\begin{array}{c}\mathbf{9 7 . 7 \%} \\
\text { High }\end{array}$ & $\begin{array}{c}\mathbf{9 9 . 9 \%} \\
\text { High }\end{array}$ \\
\hline $\mathrm{dCL} / \mathrm{dALPDEG}$ & ALPDEG & $4.72 \mathrm{E}-05$ & $2.02 \mathrm{E}-02$ & $2.03 \mathrm{E}-02$ & $2.03 \mathrm{E}-02$ & $2.04 \mathrm{E}-02$ & $2.04 \mathrm{E}-02$ & $2.05 \mathrm{E}-02$ & $2.05 \mathrm{E}-02$ \\
\hline $\mathrm{dCL} / \mathrm{dALPDEG}$ & $\mathrm{Q}$ & $4.97 \mathrm{E}-05$ & $2.02 \mathrm{E}-02$ & $2.03 \mathrm{E}-02$ & $2.03 \mathrm{E}-02$ & $2.04 \mathrm{E}-02$ & $2.04 \mathrm{E}-02$ & $2.05 \mathrm{E}-02$ & $2.05 \mathrm{E}-02$ \\
\hline $\mathrm{d} \mathrm{CL} / \mathrm{dALPDEG}$ & ALPDEG+Q & $6.85 \mathrm{E}-05$ & $2.02 \mathrm{E}-02$ & $2.02 \mathrm{E}-02$ & $2.03 \mathrm{E}-02$ & $2.04 \mathrm{E}-02$ & $2.04 \mathrm{E}-02$ & $2.05 \mathrm{E}-02$ & $2.06 \mathrm{E}-02$ \\
\hline $\mathrm{CL}$ & ALPDEG & $2.04 \mathrm{E}-03$ & $3.95 \mathrm{E}-01$ & $3.97 \mathrm{E}-01$ & $3.99 \mathrm{E}-01$ & $4.01 \mathrm{E}-01$ & $4.03 \mathrm{E}-01$ & $4.05 \mathrm{E}-01$ & $4.07 \mathrm{E}-01$ \\
\hline $\mathrm{dCL} / \mathrm{dQ}$ & ALPDEG & $4.97 \mathrm{E}-05$ & $2.39 \mathrm{E}-02$ & $2.40 \mathrm{E}-02$ & $2.40 \mathrm{E}-02$ & $2.41 \mathrm{E}-02$ & $2.41 \mathrm{E}-02$ & $2.42 \mathrm{E}-02$ & $2.42 \mathrm{E}-02$ \\
\hline $\mathrm{dCL} / \mathrm{dQ}$ & $\mathrm{Q}$ & $1.23 \mathrm{E}-03$ & $2.04 \mathrm{E}-02$ & $2.16 \mathrm{E}-02$ & $2.28 \mathrm{E}-02$ & $2.41 \mathrm{E}-02$ & $2.53 \mathrm{E}-02$ & $2.65 \mathrm{E}-02$ & $2.78 \mathrm{E}-02$ \\
\hline $\mathrm{dCL} / \mathrm{dQ}$ & ALPDEG+Q & $1.23 \mathrm{E}-03$ & $2.04 \mathrm{E}-02$ & $2.16 \mathrm{E}-02$ & $2.28 \mathrm{E}-02$ & $2.41 \mathrm{E}-02$ & $2.53 \mathrm{E}-02$ & $2.65 \mathrm{E}-02$ & $2.78 \mathrm{E}-02$ \\
\hline $\mathrm{CL}$ & ALPDEG & $2.41 \mathrm{E}-03$ & $3.94 \mathrm{E}-01$ & $3.96 \mathrm{E}-01$ & $3.98 \mathrm{E}-01$ & $4.01 \mathrm{E}-01$ & $4.03 \mathrm{E}-01$ & $4.06 \mathrm{E}-01$ & $4.08 \mathrm{E}-01$ \\
\hline $\mathrm{CL}$ & TOTAL & $3.15 \mathrm{E}-03$ & $3.91 \mathrm{E}-01$ & $3.95 \mathrm{E}-01$ & $3.98 \mathrm{E}-01$ & $4.01 \mathrm{E}-01$ & $4.04 \mathrm{E}-01$ & $4.07 \mathrm{E}-01$ & $4.10 \mathrm{E}-01$ \\
\hline
\end{tabular}

14

American Institute of Aeronautics and Astronautics 


\section{Uncertainty Propagation in the NASA LaRC Conceptual Design Shop (CDS)}

In the past five or six years, the aeronautics group within SACD has partnered with the Aerospace Systems Design Laboratory (ASDL) at the Georgia Institute of Technology for some of its risk analysis needs ${ }^{30-33}$. The most relevant examples are the technology assessments (described subsequently), technology forecasting and technology metrics tracking ASDL performed on a yearly basis for the former Ultra Efficient Engine Technology (UEET) project, and for the more recent Vehicle Systems Program (VSP). As a parallel effort to that at Georgia Tech, a large multidisciplinary team was formed at NASA LaRC to develop the Conceptual Design Shop (CDS) as a framework in which one could "efficiently and confidently design and assess atmospheric vehicle concepts and advanced technologies to meet NASA's aeronautics goals." [CDS Scope Document]. It was of special interest to handle unconventional configurations and advanced technologies for which previous data and experience is lacking. Because uncertainty estimates of S\&C parameters (forces and moments and their derivatives) are normally requested by most control law designers, the stability and control sub-team within CDS first recognized and advocated for developing an in-house uncertainty management and risk analysis capability within CDS. This advocacy led to the development of a new Matlab-based tool called the Confidence Module (CM $)^{34}$.

The CM was to provide the framework and tools for uncertainty management, i.e. quantification, propagation, decomposition and accommodation, in the analysis and design of aircraft at the conceptual level. It was of special interest to support multidisciplinary variable-fidelity analysis and design environments, and to enable trade-off studies evaluating system performance, robustness and reliability. The five year plan was to create a tool which was capable of performing uncertainty quantification, propagation, inverse design, decomposition and robust design. Unfortunately, CDS was only funded for two years, and only the uncertainty quantification and propagation capabilities were actually implemented.

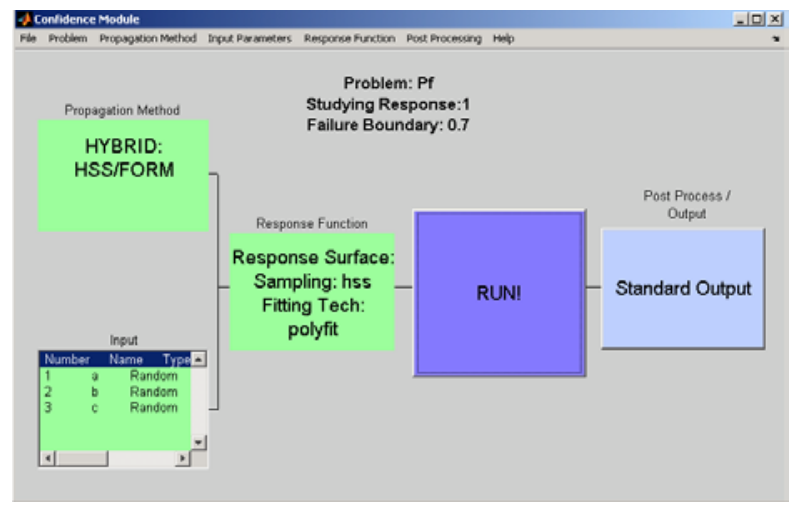

Figure 14. CM Graphical User Interface

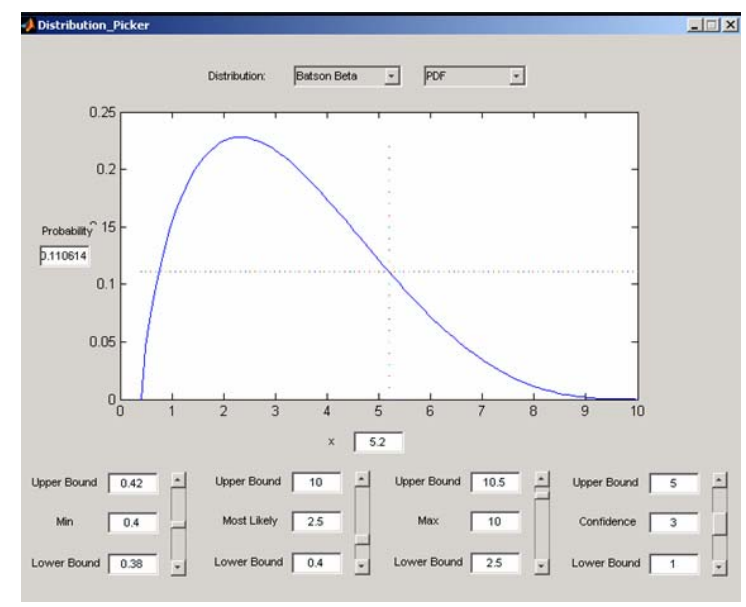

Figure 16. PDF and CDF GUI

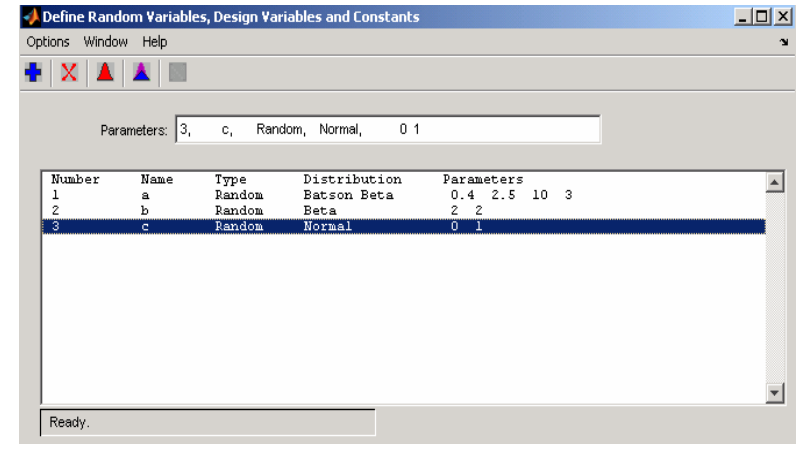

\section{Figure 15. Uncertainty Quantification for Input} Parameters

Figure 14 shows the main CM graphical user interface, written in Matlab $^{\circledR}$. The display is like a flowchart displaying the steps the user has to take to solve a probabilistic analysis problem. The bottom left box, is the uncertainty quantification box, where the input parameters and their associated probability distributions are defined. The top left box is where the user chooses the uncertainty propagation method he/she wishes to use. Finally, the two boxes link to a third box, which is where the user chooses whether he/she wants to run the analysis code itself, or run a response surface representation of it. Once the three are defined the "run" button can be clicked and the output is in the form of a "probability of failure" metric. The user defines ahead of time what is the target or maximum threshold for the response of interest, and the $\mathrm{CM}$ calculates, what the probability is of exceeding that threshold. 
Figures 15 and 16 above show the uncertainty quantification GUIs. Figure 15 allows the user to add an infinite amount of uncertain input parameters and Fig. 16 allows the user to pick the appropriate PDF or CDF describing each uncertain parameter. The distribution choices contain all Matlab ${ }^{\circledR}$ statistical toolbox distributions (beta, normal, uniform, weibull, chi-square, etc), and three customized ones which include the triangular distribution, a generalized beta, and the Robert Batson ${ }^{35}$ distribution, mentioned above.

The uncertainty propagation methods were coded in Matlab ${ }^{\circledR}$, and include sampling techniques (Monte Carlo simulations, Hammersley Sequence Sampling and Latin Hypercube), a first order reliability method (FORM), and a hybrid method, which uses the HSS sampling combined with the FORM technique, and uses the appropriate one depending on the magnitude of the probability of failure.

The response surface method currently included in the CM is a polynomial fit, and provides actual versus predicted as well as response versus individual input parameter plots, in order for the user to verify whether the fit is good. The CM has not been used for any real world problems yet, but has been applied to several sample problems, and has proven to be validated.

\section{Uncertainty Decomposition}

Uncertainty decomposition is the process of allocating rolled-up output uncertainties back to significant, primary sources. This process usually involves some kind of sensitivity analysis, which may be performed manually by trade studies, or by automated tools, such as the ADIFOR 3.0 tool $^{36-41}$ which takes standard Fortran code and a specification of independent and dependent variables to produce direct (forward-mode) or adjoint (reverse-mode) Fortran code that is compiled and executed to evaluate the gradient and/or Hessian matrices of the simulation code, in lock-step with the function evaluation.

NASA's Vehicle Systems Program in 2004-2005, and other NASA projects such as the Ultra Efficient Engine Technology ${ }^{33-34}$ in the past, have asked Georgia Tech's ASDL to help with quantifying the uncertainty and its impact, in their technology assessments. Technology assessments are performed to determine if the technologies invested in, will support and meet the project goals, and to identify which technologies have the greatest impact on those goals. The assessment provides useful information to enable technology portfolio management and budget allocation decision making.

The assessment is traditionally performed by modeling a baseline vehicle and changing a few parameters to reflect the impacts of a particular technology. The process is repeated for multiple technologies individually, and then combined, provided that they are compatible. The impacts of each technology are gathered through interviews with the researchers and technologists who are familiar with the technology of interest. The results of the interview, or technology audits, are then translated into appropriate parameters within the analysis model. ASDL calls these parameters, technology k-factors or technology metrics. Each technology can therefore be modeled in the analysis code, as a vector of k-factors. However, the values for these metrics are uncertain. They are based on estimating methods of varying levels of fidelity, sometime even based on the technologist's "best guess". Hence, in order to capture this uncertainty, the technology audit process asks the technologist to provide four values for each technology metric: minimum, most likely and maximum value, as well as the confidence in the estimate, on a scale from one to five. The four values are then translated into a probability density function (PDF), using a technique proposed by Dr. Robert Batson ${ }^{35}$, as shown in Fig. 17.

The Georgia Tech ASDL uncertainty analysis is based on three steps, uncertainty quantification, propagation, and decomposition. The translation of the technologists inputs into a beta distribution constitutes the uncertainty quantification step. Now that the uncertainty in the inputs is quantified, the next step is to estimate how this uncertainty propagates through the analysis model, and how it

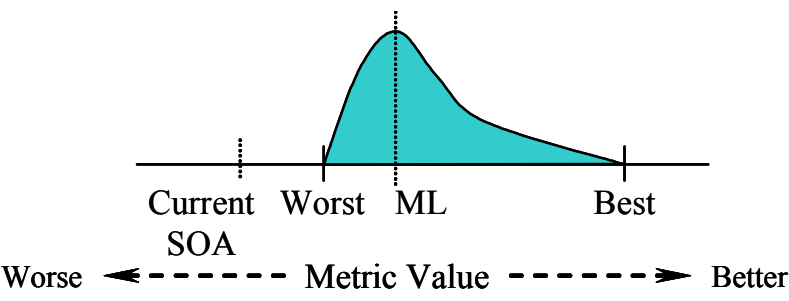

Figure 17. Probability Density Function affects the uncertainty in the results. This is performed by running a sampling technique called Monte Carlo Simulations, using the commercial tool Crystal Ball ${ }^{\circledR}$. However, Monte Carlo simulations require numerous runs and can take the analysis code days to run. Hence, instead of propagating the uncertainty through the analysis code itself, the Monte Carlo simulations are performed on response surface models of the analysis code. Each response of interest (Takeoff gross weight, fuel consumption, etc.) is expressed as a function of k-factors, in the form of a quadratic model as shown in Eq. (5). 


$$
R=b_{0}+\sum_{i=1}^{k} b_{i} k_{i}+\sum_{i=1}^{k} b_{i i} k_{i}^{2}+\sum_{i=1}^{k-1} \sum_{j=i+1}^{k} b_{i j} k_{i} k_{j}
$$

Figure 18 shows a schematic of the process. Each technology, 1, 2 and 3 have a vector of $\mathrm{k}$-factors, representing its impacts, with quantified uncertainty, on the whole vehicle. These technologies are compatible and evaluated together. The impacts and uncertainties are modeled and propagated through the vehicle's mission, and performance results are computed with its associated uncertainty. In Fig. 18, the response is shown in the form of a cumulative distribution function, which lets the user read off what confidence there is associated with a particular value.

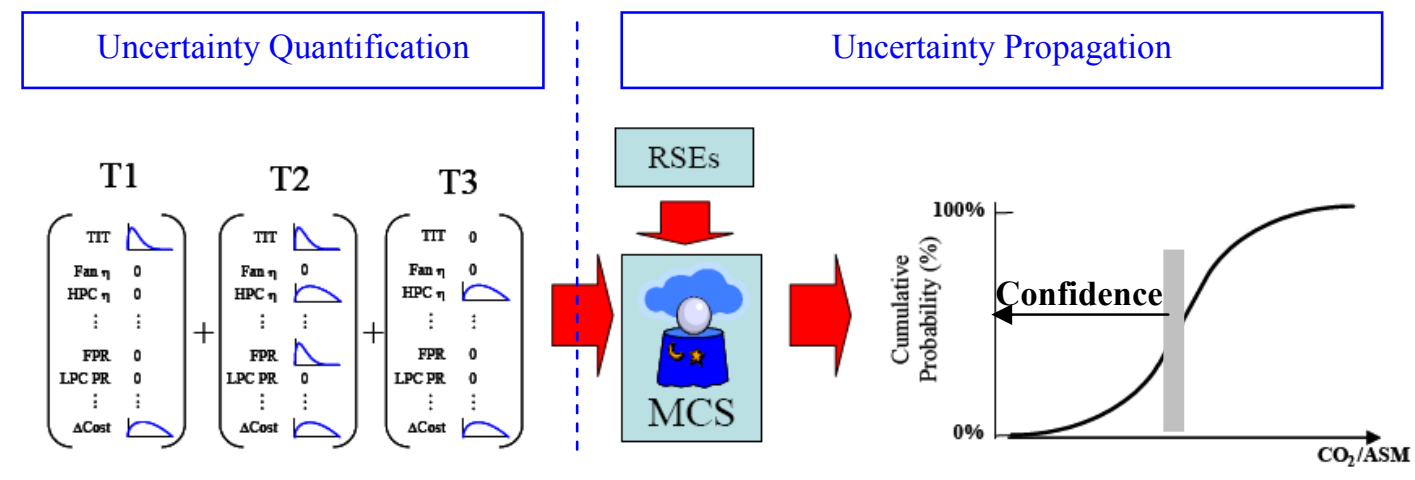

Figure 18. Probabilistic Assessment of a Technology Combination

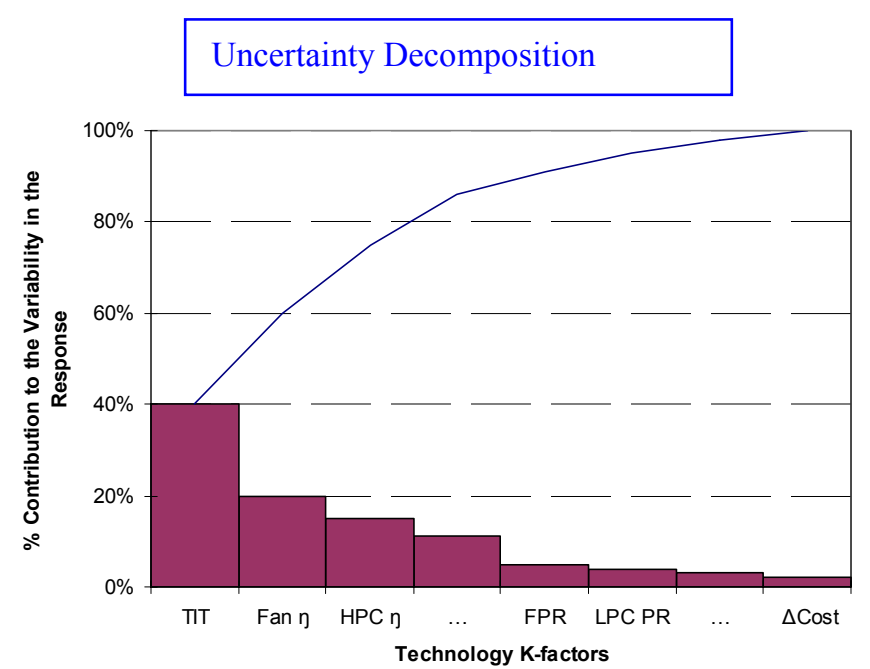

Figure 19. Pareto Analysis

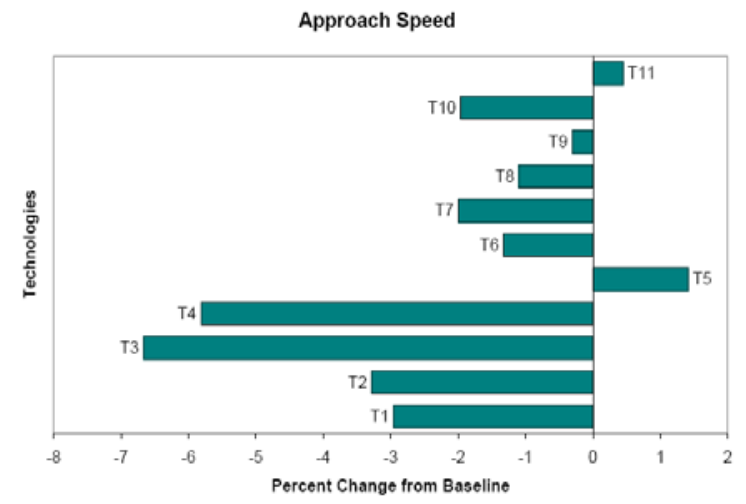

The advantage of using Response Surface Methods is that you obtain many different sensitivity and statistical data in the process of finding a good fit to the analysis model. For instance, the commercial statistical package $\mathrm{JMP}^{\circledR}$, used to generate the response surfaces allows the user to view a Pareto plot, which shows which inputs contribute to the variability in the response and by how much. This constitutes uncertainty decomposition. Therefore if the user's response is not satisfactory, the confidence is too low or the variability is too high, then the user can determine which inputs to focus on, in attempting to reduce the uncertainty in the problem. The generic Pareto plot in Fig. 19 illustrates that the response's variability is mainly due to the uncertainty in the TIT, Fan efficiency and HPC efficiency parameters. More knowledge on those three input estimates would help reduce the uncertainty in the response.

Once all the technologies are modeled individually and combined, the user obtains a comparison between the various technologies and how they contribute to the vehicle's performance, and project goals, as shown in Figure 20.

Figure 20. Sensitivity Studies and Technology Assessments 


\section{Uncertainty Management}

Uncertainty management is the multidisciplinary process of "living with" or accommodating identified uncertainties. In many ways this is somewhat like the use of safety factors, which have been historically incorporated into good engineering analysis and design practices since humans began to build thing that could fail. The difference is that with safety factors, it is difficult or impossible to quantify how changing an analysis or design variable affects the margin of safety, whereas the goal of uncertainty analysis, propagation, decomposition, and management is specifically to quantify these effects. Uncertainty analysis may be incorporated into analysis and design results by providing a range of possible answers or a confidence interval to decision makers and stakeholders, with all the relevant assumptions and modeling limitations clearly documented, rather than simply presenting single valued answers. The goal of uncertainty management should be to provide to the decision maker as much visibility into the limitations and risks inherent in the analysis or design process as possible, so that he or she would come to the same technical conclusions as the analyst, given all the relevant data and assumptions. This moves the decision risk from the analyst to the decision maker or stakeholder ${ }^{42}$. It is significant to note that in some cases, if uncertainty information can be obtained, and is not presented, that the company, decision maker, and/or the analyst might be charged with the equivalent of negligence. Therefore it is incumbent upon decision makers to request uncertainty information for results presented to them.

\section{Uncertainty Management in Multidisciplinary Design Optimization}

For the purposes of this discussion, Multidisciplinary Design Optimization (MDO) $)^{43-44}$ refers to the part of the total design process that can be formulated as an optimization problem. MDO problems usually start out as collections of autonomous disciplinary analyses with diverse data formats and diverse levels of fidelity and uncertainty in the models that describe the constituent disciplines. The autonomy, complexity, and diversity present a major challenge for the integration of disciplines into a nonlinear programming problem statement, quantifying various types of uncertainty associated with the MDO models and process, propagating the uncertainty and managing it in the design process. We describe several efforts aimed at managing uncertainty in multidisciplinary design.

The development of analytically founded approaches to MDO problem formulation is important in deterministic MDO because in realistic MDO environments, it is often difficult to determine a priori whether a chosen problem formulation will produce satisfactory results. Reconfiguration of the problem may be required, but the expense and complexity of integration usually leaves no room for experimentation with alternative formulations. The difficulty is exacerbated with the need to manage uncertainty, because the presence of uncertainty always increases computational expense. This has motivated an ongoing effort in building a systematic analytical foundation for MDO problem synthesis and solution.

The effort was first focused on the analysis of existing MDO formulations. The analysis and the attendant numerical demonstrations revealed the direct influence of MDO problem formulation on computational tractability of the resulting optimization problem ${ }^{45-48}$. The analysis clarified why and how all MDO problem formulations are related to each other through appropriate elimination of variables and constraints.

The basic relationship among all problem formulations and the fundamental difficulty in choosing an appropriate formulation a priori point to a clear need for flexible MDO problem implementation that should assist researchers and practitioners in formulating and reconfiguring MDO problems with ease, as well as in extracting information that would enable reasoning about various aspects of formulations, including the associated uncertainties, and their use in the context of analysis and optimization.

Developing such a general, flexible methodology for managing disciplinary subsystems in MDO problem formulation and solution is a feasible undertaking because the context of optimization requires a limited number of basic entities to be manipulated: design variables, objective and constraint functions obtained by evaluating the outputs of the contributing analyses, and possibly the associated derivatives. Recent developments in computational frameworks have eased various implementation aspects for simulation-based design. The mechanics of reasoning about MDO problem formulation and implementation, however, have remained elusive, possibly for reasons of difficulties in specifying MDO problems. Recent developments ${ }^{49-50}$ suggest that the complexity of MDO specification can be overcome by a linguistic, context-free, grammar-based approach to MDO problem description, formulation, and solution, called reconfigurable multidisciplinary synthesis (REMS).

REMS is a conceptual framework that comprises an abstract language and a collection of processes that provide a means for dynamic reasoning about MDO problems in a range of contexts, with assistance from computer science techniques. REMS starts with a description of disciplinary data according to the rules of a grammar. Lexical analysis 
of the description followed by linkages of multidisciplinary graphs allows the researcher to manipulate basic computational components in a number of contexts. The components can be assembled into various MDO problem formulations and solution algorithms, including hybrid strategies, with relative ease. The range of contexts for reasoning about MDO spans tasks from error checking and derivative computation to formulation and reformulation of optimization problem statements. Computational implementation of REMS is in progress.

Rigorous analysis of MDO problem formulations enables further consideration of uncertainty in MDO problems. SACD has supported the efforts of a group at Vanderbilt University in developing methods for managing uncertainty in MDO. The effort has resulted in a number of systematic approaches to reliability-based MDO and other methods for designing systems in the presence of uncertainty ${ }^{51-55}$.

\section{E. Robust Design Methods}

\section{Approximation and Model Management Optimization}

First-order approximation/model management optimization ${ }^{56-60}$ (AMMO) is a rigorous methodology for solving high-fidelity design optimization problems with minimal expense in high-fidelity function and derivative evaluation. AMMO combines the traditional engineering practice of using models of varying fidelity with state-ofthe-art nonlinear programming algorithms. It is a general approach applicable to any derivative based optimization algorithm and any combination of high-fidelity and low-fidelity simulations, e.g., the solution of the governing differential equations on meshes of varying degree of refinement (variable-resolution) or the use of a range of physics, from detailed to less accurate (variable-fidelity physics models).

In proof-of-concept demonstrations, basic AMMO algorithms have been shown to yield from three to sevenfold savings in terms of high-fidelity simulations, while attaining high-fidelity optima. The work in AMMO quickly spread worldwide, initiating related lines of research in universities, national laboratories, and industry and leading to improving or enabling tractability of single-discipline and multidisciplinary design problems. AMMO algorithms are now studied as a candidate for reducing the cost of uncertainty-based design.

The idea of AMMO is to transfer the computational load from high-fidelity models alone to low-fidelity models corrected with high-fidelity information occasionally but systematically. If the corrected lower-fidelity model does not predict the trends of the higher-fidelity model adequately, AMMO can resort to a higher-fidelity model in the available suite of models or it can take shorter low-fidelity steps before requiring high-fidelity recalibration. In the worst case, AMMO is conventional optimization with the high-fidelity model.

The use of variable-fidelity physics models presented the most intriguing demonstration. In this case, although one may expect similar global trends in some regions of the design space, there is no guarantee of similar trends, in general. For instance, in aerodynamics, when viscous and shear effects become active, the trends of low-fidelity models that do not capture such effects may differ from those of the high-fidelity models.

One of the proof-of-concept tests considered an aerodynamic optimization of a multi-element airfoil designed to operate in transonic conditions ${ }^{58}$. The transonic free-stream Mach number and the multi-element nature of the airfoil make inclusion of the viscous effects very important for obtaining physically correct results. This is confirmed in Fig. 21, which depicts the Mach number in the flow for the high-fidelity and low-fidelity models, where the boundary and shear layers are clearly visible in the viscous case. To capture the viscous effects, the governing equations of the high-fidelity model are the Reynolds-averaged Navier-Stokes (RANS) equations. The low-fidelity model is represented by the Euler equations. The analysis in both the RANS and Euler modes is provided by FUN2D ${ }^{61}$, an unstructured mesh flow solver that also provides the sensitivity derivatives via the adjoint approach. The mesh for the viscous model consists of 10449 nodes and 20900 triangles. The mesh for the inviscid model comprises 1947 nodes and 3896 triangles. The free-stream Mach number is $\mathrm{M}_{\infty}=0.75$, the Reynolds number is $\operatorname{Re}=9 \times 10^{6}$, and the global angle of attack is one degree.

Figure 22 depicts the level sets of the drag coefficient for the viscous and inviscid models. The solution for each problem is marked with a circle. The problem manifests the most adverse situation: not only is the low-fidelity model not a good approximation of the high-fidelity model but, in fact, the descent trends in the two models are reversed.

The experiments were conducted on an SGI ${ }^{\mathrm{TM}}$ Origin $^{\mathrm{TM}} 2000$ workstation with four MIPS RISC R10000 processors. Time per one low-fidelity analysis was approximately $23 \mathrm{CPU}$ seconds with attendant sensitivity taking between 70 and 100 seconds. One high-fidelity analysis took approximately 21 minutes with 21 to 42 minutes per sensitivity.

The problem with two variables was first solved with the high-fidelity model alone using a commercial solver, in order to obtain a baseline number of analyses to find an optimum. The problems were then solved with AMMO. As the time per low-fidelity computation was negligible in comparison to the high-fidelity computation, the savings were estimated strictly in terms of high-fidelity evaluations. 
Because the ability to handle high-dimensional problems is an important attribute of first-order, the experiments were repeated for the problem with 84 variables. The additional variables were represented by parameters describing the shape of the airfoil. The savings were similar to those of the two-variable case: in both cases, AMMO yielded five-fold savings in terms of high-fidelity analyses.

The analysis of the results revealed that the savings were not surprising despite the dissimilarity of the model trends. This is illustrated in Fig. 23 for the two-variable case. The plot on the left shows the level sets of the highfidelity model with the solution. The plot on the right depicts the level sets of the low-fidelity model corrected at the initial point. The initial point is marked by a square. The correction is applied to the entire region to visualize its effect: the correction, containing both the function and derivative information at the initial design, reversed the trend of the low-fidelity model, allowing the optimizer to find the next iterate in the northwest corner of the plot, marked by a circle. AMMO located the solution $\left(\alpha=1.6305^{\circ}\right.$, flap y-displacement $\left.=-0.0048\right)$ of the high-fidelity problem at the next iteration. The high-fidelity drag coefficient at the initial point, $C_{D}^{\text {initial }}=0.0171$ and at the solution, $C_{D}^{\text {final }}=0.0148$, yielded a decrease of approximately $13.45 \%$. The drag reduction of the 84 -variable case was approximately $25 \%$. Details of the demonstration may be found in Ref. 58 .

The relation of AMMO to managing uncertainty in design is two-fold. First, AMMO implicitly reduces uncertainty associated with design by enabling the use of higher-fidelity modeling at decreased cost. Second, AMMO-based ideas can be used in decreasing the cost of explicit uncertainty-based design optimization ${ }^{62}$.
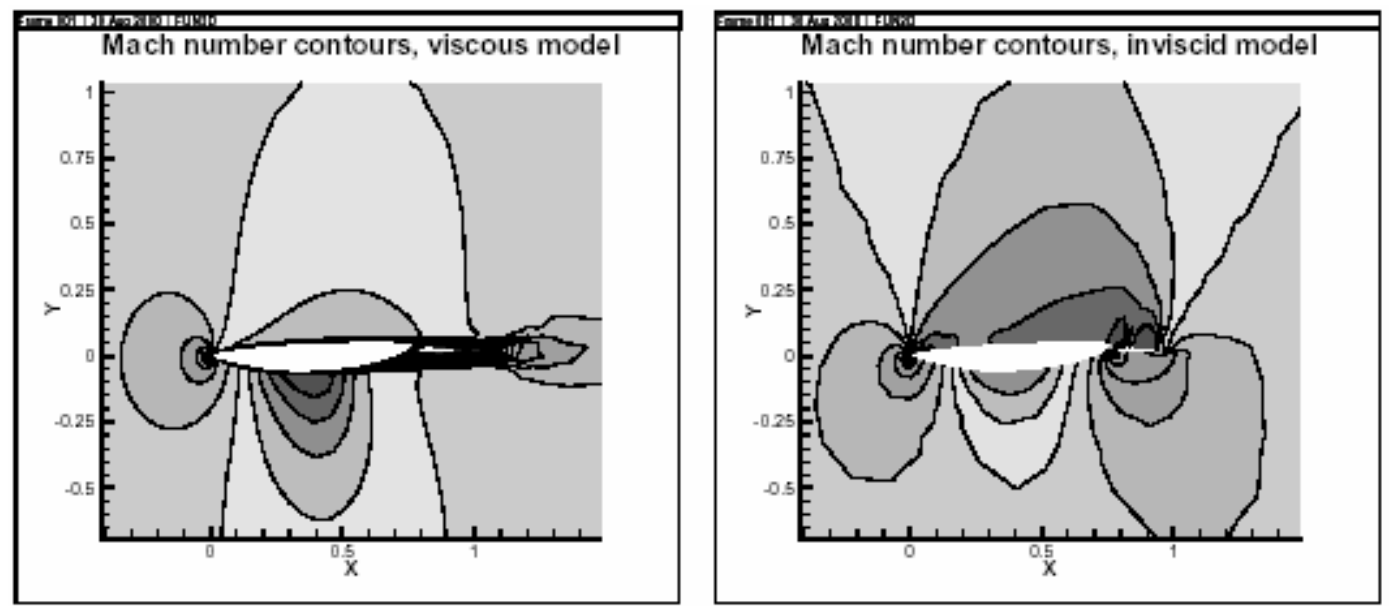

Figure 21 - Mach contours for transonic flow around an airfoil, high- and low-fidelity models.
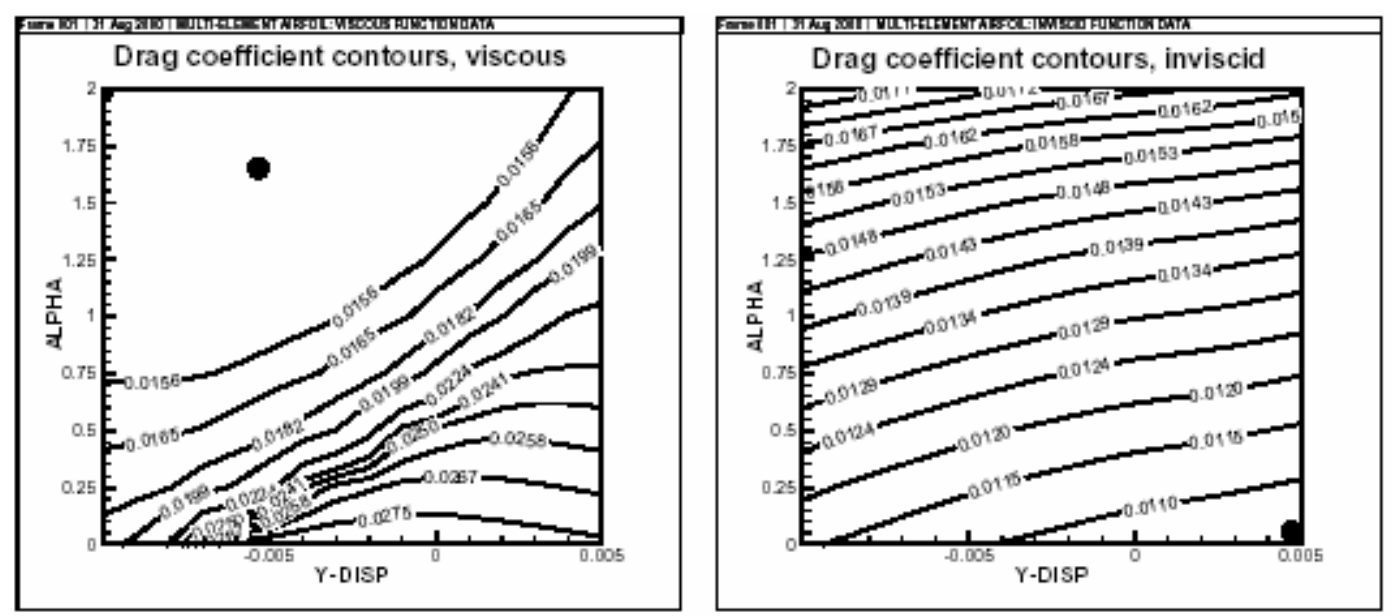

Figure 22 - Contours of objective function for transonic flow around an airfoil, high- and low-fidelity models. 

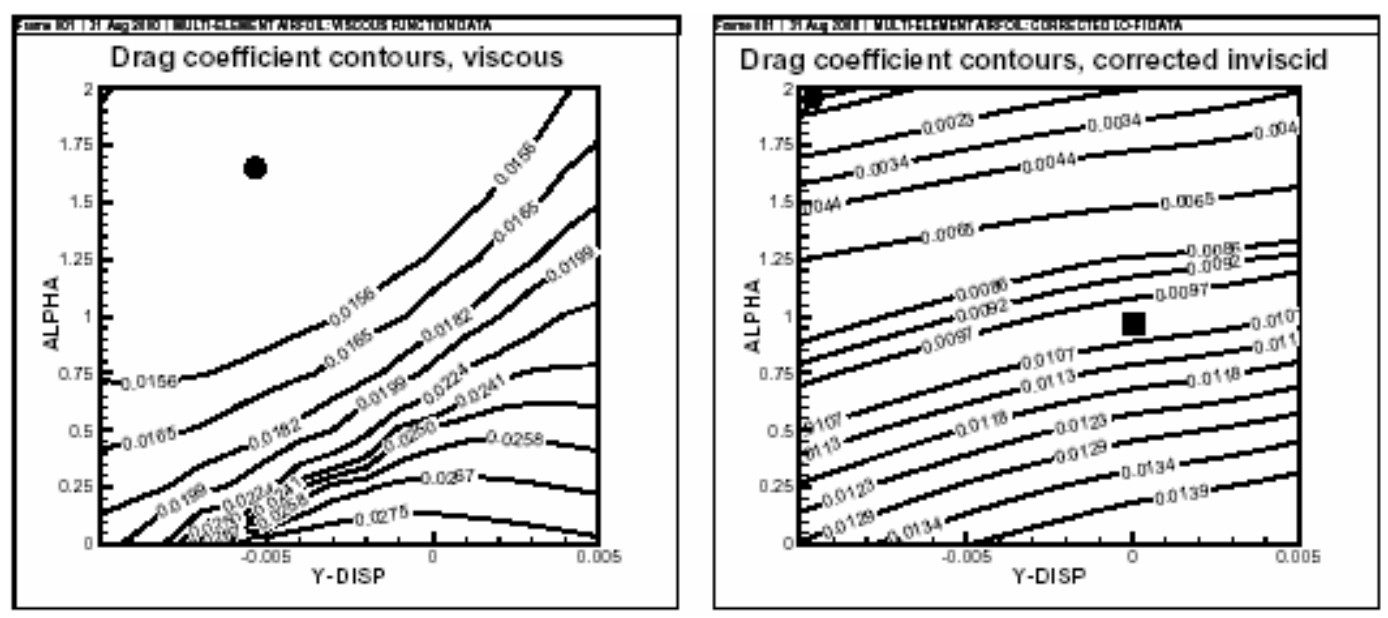

Figure 23 - Illustration of design under uncertainty using AMMO optimization using high- and low-fidelity models.

\section{Design Under Uncertainty in the Flight Optimization System (FLOPS) Code}

As described in Ref. 12, the method of moments was implemented in the FLOPS aircraft mission analysis program for several classes of statistically independent, normally-distributed, random input variables and several classes of random output variables, to enable robust design. Different levels of input uncertainty and required constraint satisfaction were imposed. The effect of uncertainty on the design point, compared with a deterministic design, is noted. In Ref. 12, the example was also analyzed and validated with a Monte Carlo simulation; additional work was performed using the simulation-search method within the UNIPASS tool.

In this study, the effects of uncertainty in two input aircraft design variables are considered for the purposes of illustrating uncertainty propagation through the FLOPS code and robust design under uncertainty. For the demonstration (and following the derivation in Ref. 11), these input variables were assumed statistically independent, random, and normally distributed about a mean value. These assumptions simplify the implementation and help quantify the input uncertainties. The assumption of the variables being statistically independent is not required; correlation between the variables can be easily accounted for within the formulation at the cost of more computational work. For non-normal input distributions, the methods of moments' corrections are only approximately correct

For simplicity, the demonstration was derived from a particular sample cases distributed with the FLOPS code, which is the of five-design variable subsonic transport design (xfp2.in). The input file was modified to allow only the variables THRUST (the maximum rated thrust per engine, in pounds force), and SW (the wing reference area, in square feet), to be active design variables; upper and lower bounds were also specified for these design variables. The modified input file was then used for both deterministic and robust optimizations. The robust optimizations had various levels of input uncertainty for the active design variables and various levels of required constraint satisfaction, both specified in auxiliary input file to FLOPS. For the example case, the specified input variable uncertainty corresponds to $5 \%$ of the mean value for each of the two input variables. Since the mean value of THRUST for this problem is $47,500 \mathrm{lb}$ and the mean value of SW for this problem is $2272 \mathrm{ft}^{2}$, the variability corresponds to $2375 \mathrm{lb}$ and $113.6 \mathrm{ft}^{2}$ for the two variables, respectively. The constraint satisfaction requirement ranged from zero (where the constraint was satisfied with $50 \%$ probability for a normal distribution) to three (constraint satisfied with $99.9 \%$ probability for a normal distribution). The optimization objective was specified to be the aircraft gross takeoff weight. The seven possible aircraft performance constraints, normally activated with this sample problem, were used. These included the aircraft required range (which were held fixed for this problem), the approach speed, the takeoff and landing distances, the approximate missed-approach and second-segment climb gradients, and the excess fuel. The Broyden-Fletcher-Goldfarb-Shano (BFGS) optimization method (the default among several optimization methods available within the FLOPS code) was used to solve this problem. In the FLOPS implementation of this optimization method, a composite objective function was minimized. The composite objective function was composed of the true objective augmented with a highly nonlinear penalty function that grew rapidly as the design variables approach their upper or lower bounds, and as constraints become active.

Figure 24 is a simplified aircraft sizing contour ("thumbprint") plot illustrating the design space near the deterministic and robust optimizations for this case. The $\mathrm{x}$-axis of the figure shows a normalized value of the maximum rated thrust per engine, ranging from 20,000 to 60,000 pounds force; the $y$-axis of the figure shows the 
normalized value of the reference wing area, ranging from 1000 to $5000 \mathrm{ft}^{2}$. The figure is simplified from a typical thumbprint plot in that only the active constraint violation boundaries for the specified problem are shown, rather than multiple contours representing various values of the limit state function. The constraint violation boundaries were interpolated from a parametric variation of the two variables with nine equally spaced points in each direction. Also shown in Fig. 24 are the locations of final design points for the deterministic and three mean robust optimizations. In this example, both FLOPS constraint 2 (the upper limit on approach speed) and FLOPS constraint 5 (the lower limit on missed approach climb gradient) were active for the deterministic optimization. As expected, the deterministic design point is found at the intersection of the two active constraints. The optimization path was almost entirely within the feasible region in the figure.

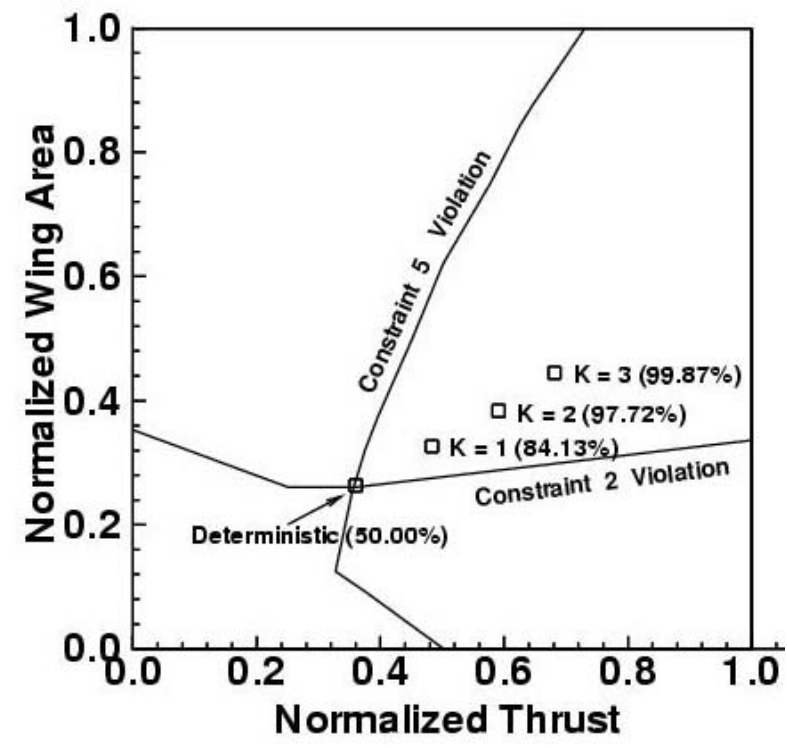

Figure 24 - Aircraft thumbprint plot illustrating robust design of a subsonic transport jet.
The three robust optimization points (labeled $\mathrm{K}=$ 1,2 , and 3 in the figure) correspond to imposed constraint satisfaction margins of 1,2 , and 3 standard deviations about the mean value of the deterministic solution. The offset in the robust design points from the constraint violation boundaries is proportional to both the imposed input uncertainty and the gradient of the constraint with respect to the uncertain design variables. Fig. 24 also shows that the robust optimization with $\mathrm{K}=$ 1 enforces a greater margin of satisfaction for both constraints than does the deterministic optimization. Similarly, each of the robust solutions enforces greater constraint satisfaction, with increasing values of $\mathrm{K}$ than either the deterministic solution or the robust optimizations with smaller values of $\mathrm{K}$. For the deterministic optimization, the constraint satisfaction with respect to single constraint violation is only $50 \%$ probability for an output normal distribution; for $\mathrm{K}=1$, this probability increases to about $84 \%$; for $\mathrm{K}=2$, the probability is about $97.7 \%$; and for $\mathrm{K}=3$, the probability rises to about $99.9 \%$.

Simultaneously (not shown in the plot), the aircraft weight increases with increased constraint satisfaction from the deterministic value of $213110.5 \mathrm{lb}$ to a value of $217052.6 \mathrm{lb}$ for $\mathrm{K}=1$, to a value of $220222.0 \mathrm{lb}$ for $\mathrm{K}=2$, and to a value of $223443.1 \mathrm{lb}$. for $\mathrm{K}=3$. The weight for $\mathrm{K}=3$ is about $5 \%$ higher than the deterministic solution.

\section{Design Under Uncertainty for Coupled Aero-Elastic Wing}

A system has been developed to couple an iterative Computational Fluid Dynamics (CFD) solver and a Finite Element Method (FEM) structural solver with gradient-based optimizers, First Order Second Moment (FOSM) uncertainty analysis, and First Order Reliability Method (FORM) analysis ${ }^{63-64}$. The system has been applied to perform deterministic optimization, robust optimization, and reliability analysis for a flexible trapezoidal wing. The unique feature of the system is that the iterative loops of the methods (Euler/Navier-Stokes CFD, coupled aero/structural interaction, quasi-analytic sensitivity analysis, Newton method-based optimization, Most Probable Point (MPP) reliability analysis) are solved simultaneously. That is, a well-converged solution to any inner iteration loop is not required - or even expected - until the outermost iterative loop reaches convergence. This feature has been shown to significantly reduce the computational expense relative to simply nested analyses. Previous papers ${ }^{63-65}$ documenting this work have compared the method to other Simultaneous Analysis and Design (SAND) methods. An important property of this method is that rather than performing all the functions in a monolithic code that would need to be validated, SASDO allows us to assemble familiar analysis tools for each part of the analysis. Quasi-analytic sensitivity analysis was enabled for the major analysis components by automatic differentiation of the source code. Several of the interface methods were easily differentiated "by hand".

The system was applied to a simple trapezoidal planform wing of dimensions similar to a commercial transport, flying at transonic speeds typical of a commercial transport. The CFD and FEM analysis methods are described more fully in the referenced papers and their references. The FE structure model was composed of spars, ribs, and skin as shown in Figure 25. The aerodynamic characteristics were modeled by a finite volume CFD solver, CFL3D. 


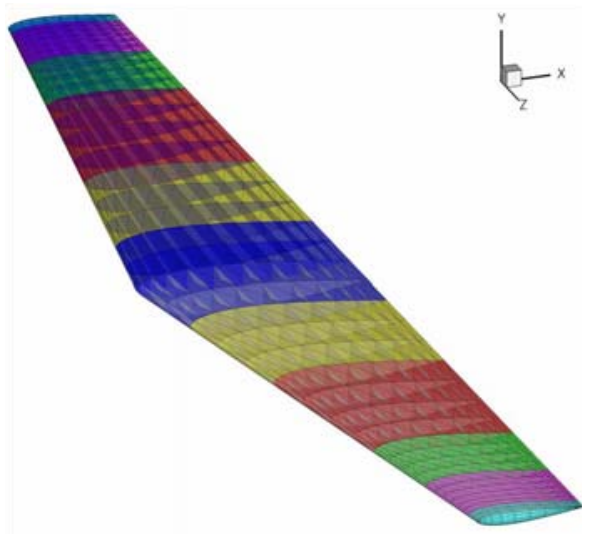

Figure 25. Finite Element structural model.

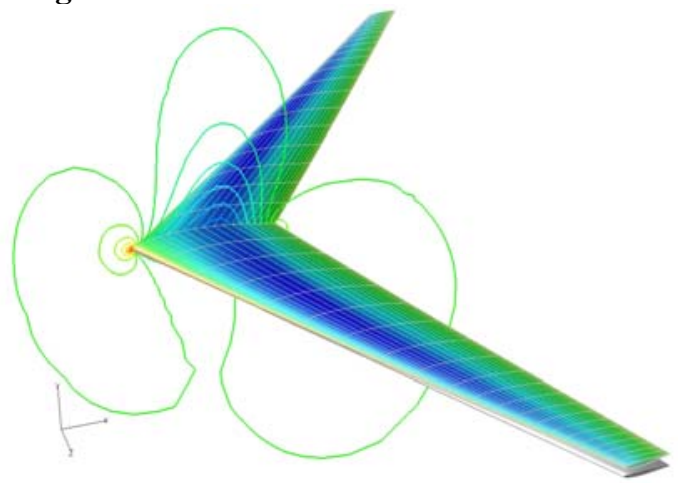

Figure 26. CFD solution (pressure contours) shown on deflected surface mesh.

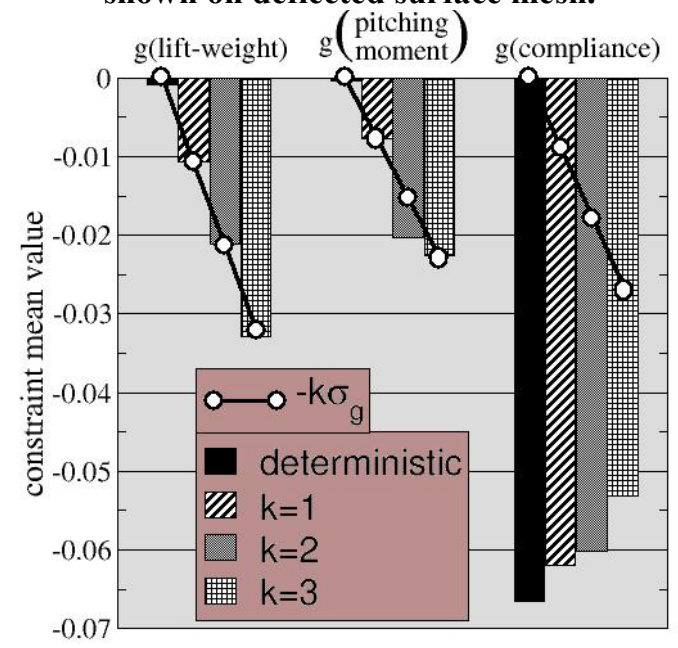

The mesh on the wing surface is shown in Fig. 26 after it has been deflected due to aerodynamic loads. Structural element sizing parameters and geometry parameters such as wingspan, airfoil maximum thickness, and airfoil camber were used as design variables in several deterministic optimizations. In order to reduce the number of parameters, the structural element sizing parameters were grouped geometrically such that one parameter attributed to each zone shown in Fig. 25 was used to control the sizes of all the elements within the zone. Uncertainty in several of those variables was propagated through the coupled analyses to conduct robust optimizations and reliability analyses. For optimization studies the objective function was typically the ratio of lift to drag. Constraints were imposed on geometric quantities such as leading edge radius, on discipline-specific quantities such as tip deflection, and on quantities using information from multiple disciplines, e.g. compliance, which is the work done by the aerodynamic loads to deflect the structure, and payload, which is the difference between the aerodynamic lift force and the structural weight. Those were also the dependent variables in the robust design and reliability analysis studies.

A comparison of some robust design results is shown in Figs. 27-29. The root airfoil thickness and camber, and the element thicknesses in the two inboard zones were considered as uncertain design parameters. A coefficient of uncertainty of 0.0001 was attributed to each. The deterministic optimization was performed first. Then several robust optimizations were performed using the deterministic result as the initial design. The optimizations were conducted accounting for several levels of uncertainty. The uncertainty effects on the optimization process are most notable in the way the constraints are satisfied as shown in Figure 27. For robust design using a FOSM method, the deterministic inequality constraint, $g \leq 0$, is met with some desired probability by representing it as $\bar{g}+k \sigma_{g} \leq 0$, where $\bar{g}$ is the mean value of the constraint, $\sigma_{\mathrm{g}}$ is the standard deviation of the constraint determined by propagating the uncertainties in the uncertain variables, and the parameter $k$ determines the probability of satisfying the constraint. An increase of the parameter $\mathrm{k}$ indicates an increase in the specified target probability of satisfying the constraints. Assuming a normal Gaussian distribution of the output variables, values of $k=1$, 2 , and 3 would represent probabilities of $84.13 \%, 97.73 \%$ and $99.87 \%$, respectively. In Fig. 27 , the bars show the mean Figure 27. Effect of uncertainty on constraints. value of the constraint functions, while the circles show the
offset required to meet the constraint with the desired probability. The three constraints shown are the difference between the aerodynamic lift force and the structural weight, the compliance, and the pitching moment coefficient. The constraints as shown in the figure have been scaled. Figure 28 shows the changes in the design variables, the mean values of the parameters, resulting from the deterministic and robust optimizations. The optimizer tends to reduce the root thickness to decrease the shock strength, thereby reducing the drag and increasing the ratio of lift to drag until the constraints become active. Increased target probability of satisfying the payload constraint requires the structural weight to decrease and/or the lift to increase. Reduced element sizes reduce the weight but allow increased 


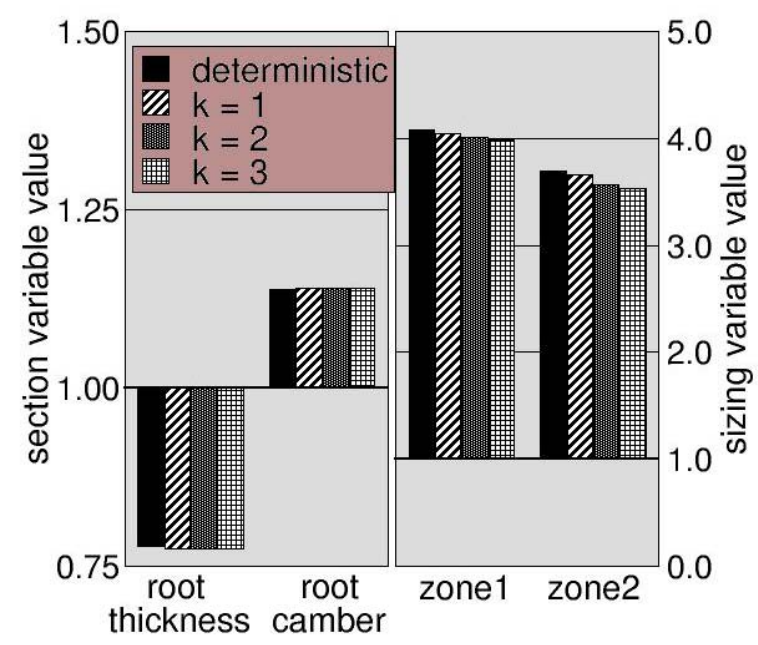

Figure 28. Effect of uncertainty on design variables.

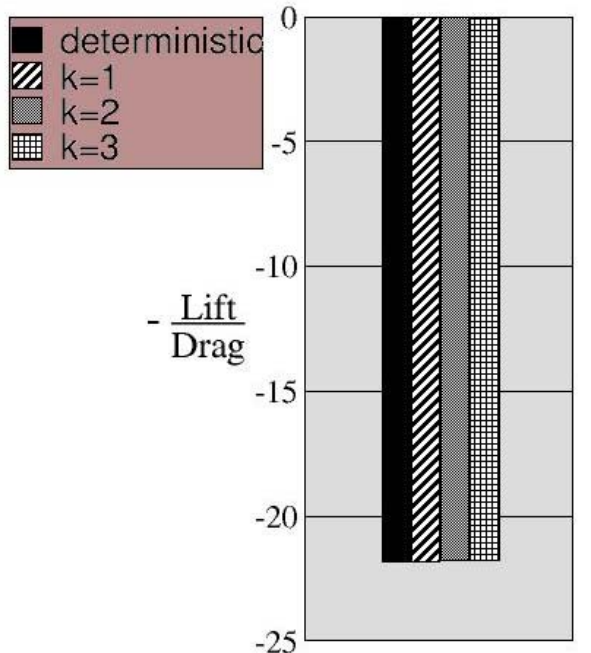

Figure 29. Effect of uncertainty on objective function.

bending and twist. The twist is exhibited in the form of washout, which helps to reduce the pitching moment and increase the probability of meeting that constraint, but it also decreases the lift. The root airfoil camber is increased to offset the reduced lift. The optimizer balances these conflicting tendencies. Figure 29 shows that, in the neighborhood of these design values, the objective function, the ratio of lift to drag, is relatively insensitive to the design variable changes necessary to satisfy the constraints.

\section{F. Reliability Analysis Methods}

An unconventional application of reliability methods is now described; more conventional reliability assessments are discussed in Ref. 6. Reliability assessments were performed for the results of the aero-elastic robust designs (described previously in section E3) using three FORM approaches: the Hasover-Lind-Rackwitz-Fiessler (HL-RF) Reliabilty Index approach (RIA), the Performance Measure Approach (PMA) and a PMA-based RIA (PRIA) ${ }^{65}$. A fundamental difference between the FOSM robust design assessment of uncertainty and the FORM reliability assessment of uncertainty is where in the design space the functions are evaluated. The uncertainty for robust design is characterized by information evaluated at the mean value of the output function; whereas, the uncertainty for reliability assessment is characterized by determination of a Most Probable Point (MPP) and evaluation of the constraint function at that point. The MPP can be determined by an optimization process. The RIA approaches produce a reliability index $\beta^{*}$ that, similarly to the parameter $k$ used in the robust design process, corresponds to a probability of satisfying the constraint. The performance measure $g_{p}^{*}$ is the offset from the constraint (limit-state function) that will result in the target reliability. A detailed description of mathematical basis and the solution methods for the three approaches are described in Ref. 63 and its associated references. Table 3 shows a comparison of the reliability assessment parameters and the robust design parameters. For the RIA results, the comparison can be drawn between the reliability index $\beta^{*}$ and the target $k$ specified in the robust design. For the PMA results the comparison can be drawn between the performance measure $g_{p}^{*}$ and the target constraint value $\bar{g}+k \sigma_{g}$ used for the robust design. The reliability analyses are consistent with each other; but, the agreement with the robust design seems to degrade with increasing values of the robust design target probability $P_{t}$. Differences in the results of the two methods can come from several sources. The uncertainty for both the robust design method and the Reliability Assessment methods is characterized using only first order information. Although the input parameter uncertainty is characterized by a Normal distribution, for a non-linear function such as those resulting from the coupled aero/structural analysis, there is no expectation that the output distribution is also Normal; but, the parameters being compared are derived on the assumption that it is. As described earlier, the uncertainty information for the robust design is evaluated at the mean value, which more accurately characterizes the center portion of the distribution, whereas, the uncertainty information for the reliability assessment is evaluated at the MPP, which more accurately characterizes the tails of the distribution. In addition to the mathematical basis for differences, the processes for the robust design and the reliability assessment are iterative methods, as are the underlying 
multidisciplinary analysis methods, so convergence tolerances for those iteration loops have potential for error. But the differences for all the cases shown are relatively small.

Table 3. Comparison of Reliability Assessments of Robust Designs.

\begin{tabular}{|c|c|c|c|c|c|c|c|c|}
\hline \multirow{3}{*}{ Constraint } & \multirow{3}{*}{$\begin{array}{l}\text { PMA } \\
g_{p}^{*} \\
\end{array}$} & \multirow{2}{*}{\multicolumn{3}{|c|}{ Robust Design }} & \multicolumn{4}{|c|}{ Reliability Index Approach } \\
\hline & & & & & \multicolumn{2}{|c|}{ HL-RF } & \multicolumn{2}{|c|}{ PRIA } \\
\hline & & $\bar{g}+k \sigma_{g}$ & $k$ & $P_{f}$ & $\beta$ & $1-P_{f}$ & $\beta$ & $1-P_{f}$ \\
\hline$g(L-W)$ & -0.00219 & 0.00005 & 1 & 0.8413 & 1.206 & 0.8862 & 1.199 & 0.8848 \\
\hline$g(L-W)$ & 0.00122 & 0.00031 & 2 & 0.9773 & 1.879 & 0.9699 & 1.859 & 0.9685 \\
\hline$g(L-W)$ & 0.00017 & -0.00075 & 3 & 0.9987 & 2.983 & 0.9986 & 2.939 & 0.9984 \\
\hline$g\left(C_{m}\right)$ & -0.00019 & -0.00003 & 1 & 0.8413 & 1.038 & 0.8504 & 1.050 & 0.8531 \\
\hline$g\left(C_{m}\right)$ & -0.00873 & -0.00500 & $2^{*}$ & 0.9773 & 3.471 & 0.9997 & 3.586 & 0.9998 \\
\hline$g\left(C_{m}\right)$ & -0.00695 & 0.00045 & 3 & 0.9987 & 4.164 & 1.0000 & 4.165 & 1.0000 \\
\hline
\end{tabular}

* Constraint active, but not tight

\section{Risk Analysis / Management}

This past year, FY2006, SACD worked with NASA's Exploration Systems Mission Directorate, (ESMD) to define how this Directorate will utilize the concept of Continuous Risk Management (CRM) ${ }^{2}$ to assist in managing the wealth of activities which must occur to fulfill the nation's space exploration vision. This "Vision for Space Exploration" (http://www.nasa.gov/pdf/55583main_vision_space_exploration2.pdf) is broad, dynamic and spans the technological gamut from utilization of State-of-the-Art technology, to future integration of technologies which currently may only be laboratory investigations. The Vision provides for the milestones of replacing NASA's current space shuttle system with a Crew Exploration Vehicle (CEV) which initially has docking access to the International Space Station (ISS). A launch vehicle, Crew Launch Vehicle (CLV) is also being designed to place the CEV into Earth Orbit. Heavier cargo is going to be delivered by another launch vehicle, the Cargo Launch Vehicle, $(\mathrm{CaLV})$. As confidence in the CEV system grows, mission goals will start to include requirements for Lunar surface access and science and technology demonstration activities. Additional flight elements such as an Earth Departure Stage, Robotic Elements, and a Lunar Surface Access Module, will be required. Again as confidence in NASA's ability to function in a lunar environment grows, mission goals will move on and push for the same type of exploration activities to occur on the Martian surface. Beyond the activities envisioned for Mars and depending upon the success of the Vision's objectives, activities which reach ever further into the space environment may be pursued.

The long term success of NASA's exploration initiative also requires that it be performed in a safe, productive, and cost controlled manner. To formalize the Safety and Program Management activities which must be integrated across all of the above activities NASA has chosen to implement CRM with tight control via utilization of the commercially available Risk Management computer program, Active Risk Manager (ARM) ${ }^{66}$. For a project of this magnitude CRM is mandated by NASA Policy Regulations "NASA Program and Project Management Processes and Requirements", and "Risk Management Procedural Requirements" ${ }^{2}$. Active Risk Manager brings to the table a process tailorable computerized database for Risk Management. Client access to the database requires only a personal computer with web access and an account in NASA's single sign on collaborative engineering environment ICE, Integrated Collaborative Environment. NASA's CRM process is mapped to the ARM application to provide all users in ESMD and its sub-tier organizations, including contracting organizations, a consistent interface to risk data no matter which parent organization is being directly supported. Tight control in this case implies that certain levels of management, Directorate/Program/Project, each incorporate ARM into their Risk Management process. Certain report formats available to all ARM users can be used to report RM information in a consistent manner throughout the organizational hierarchy. A standardized " $5 \times 5$ " Risk Classification matrix is prescribed and the three 
levels of impact, low, moderate and high are mapped to this classification matrix. RM tool and reporting requirements and other "Tight Control" procedures do not however try to dictate to such a fine grain that RM information becomes un-informative at the lowest organizational tier level where risk management is actually occurring. Risk managers are free to tailor terms in the definition of their risks such that they are handled by appropriate resources. ESMD recognizes that effective risk management occurs when mitigation activities are delegated to the organization element closest to the problem. Risks at lower levels but of great consequence are reported up to higher level organizations and the ARM database facilitates this process.

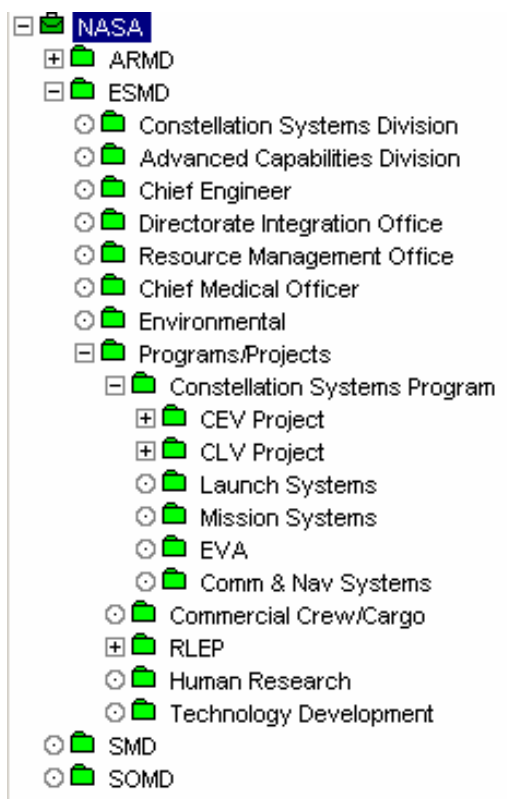

Figure 30. ESMD Risk Breakdown Structure

Effective Risk Management across a multiorganizational hierarchy is dependent upon efficient and unfettered access to data by all stakeholders. ARM provides for user definition of a hierarchical structure of risk data. In ESMD's case, as illustrated in Fig. 30, this hierarchy is based upon both the Organizational Breakdown Structure (OBS) and within organizations the Work Breakdown Structure of particular tasks (WBS). ARM access is provided on a read-only basis to practically any person involved in supporting ESMD's goals and objectives. A typical user can see all risk data within the database, not just data within their parent organization. This physically facilitates the concept of Risk Communication and fosters an attitude of collaboration, as discussed in Ref. 7 as a desirable attribute of corporate culture for NASA to nurture.

Concurrent with the rollout of ESMD's Risk Management and incorporated in that organization's Risk Management Plan, is the inclusion of Knowledge Management activities. NASA has historical data from several programs where knowledge and "Lessons Learned" have been formally gathered. The ARM data structure is also being utilized as a repository for consolidation of this data. Providing quick access to lessons learned information is intended to assist other ESMD-ARM users who may be wondering what type of risks their program may incur, and may provide historical mitigation success and failure data. To further facilitate communication between organizationally and geographically disperse ESMD team members, Communities of Practice (CoP) have been or are being set up in the following areas:

- Risk \& Knowledge Management

- Probabilistic Risk Assessment

- Strategic Analysis

- Earned Value Management

- Technology Protection

- Systems Engineering \& Integration

- Research and Technology

Focus for these CoPs are on activities related to ESMD's objectives, but it is also a web location where more general discipline dependent information can be shared. Another Knowledge Management activity encouraged by ESMD is the use of "Pause and Learn" (PAL) events. A PAL is modeled after the Army process entitled "After Action Review". At the end of selected milestones along the life-cycle of project activities, recent participants pause and have a meeting to discuss what is going right and what is going wrong with what has gotten them to the state they are currently in. The meeting is chaired by an independent facilitator and participants are free to bring up all issues for discussion and logging into meeting minutes. Such reflection hopes to celebrate what is currently going right on a project and alert participants to an agreed upon area which may require a change in approach to manage properly.

One forward looking point which NASA will emphasize in future upgrades to its ESMD Risk Management procedures is the incorporation of "Opportunity" as well as the traditional "Threat" type of Risk data. The Vision for Space Exploration can and should inspire new technologies to more efficiently meet its goals as time progresses. 
The Risk Management process can formally track Opportunities as it currently does Threats, and communicate that information across the dispersed program management hierarchy. This additional exposure of technologies to programs and program activities to technology developers is envisioned to create a working level synergism between the activities of these two groups and their managers.

Finally an effective RM activity has to integrate with other project data such as cost/schedule/resource (project management) data, and product requirements, concepts of operations and element functionality (systems engineering) activities. Within the ICE environment will be the Systems Engineering computer program, $\mathrm{CRADLE}^{67}$, and the Project Management computer program, Primavera ${ }^{68}$. Task requirements in Primavera are envisioned to become part of the Risk Activity breakdown data hierarchy in ARM. Cost and schedule impacts from Risk Management activities will be linked to the ESMD Primavera database enabling managers to assess programmatic risk impacts. Earned Value data for a project can now also include Risk data and give managers additional quantified decision making power. If project management (Primavera) data is linked to the ARM installation, Risk Managers can utilize the ARM uncertainty analysis feature to assess the variability of cost and schedule parameters for risk activities in terms of their impact on overall program planned cost and schedule allocations.

\section{A. Risk Classification:}

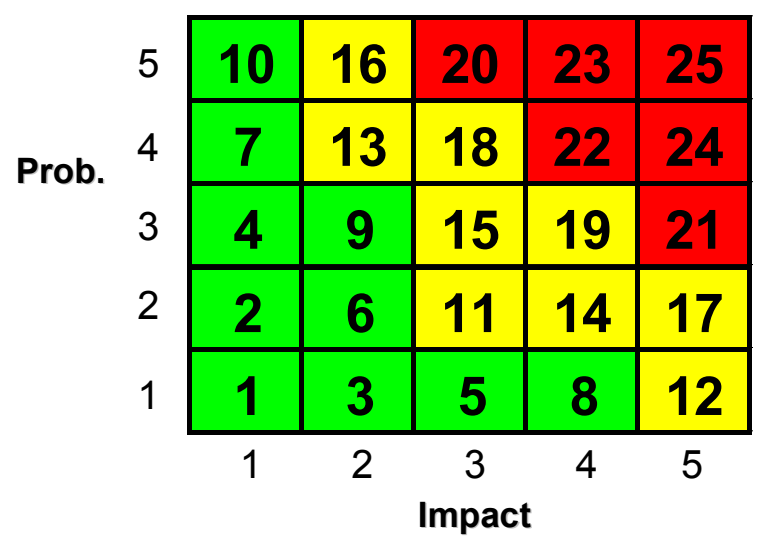

Figure 31 - ESMD Risk Classification Matrix

Reference 2 specifies in accordance with CRM techniques that a Risk Classification system be invoked. This system shall provide a means for analyzing a risk in the sense that the Probability of a risk's occurrence is rated, as is also the risk's Impact if it were in fact to occur. ESMD has standardized the Probability-Impact Risk Classification Matrix as shown in Figure 31. Low (green), Medium (yellow), and High (red) risk severity areas are indicated in the figure. In order to place a risk in this classification system a "scoring scheme" is required to assist the risk analyst with mapping the severity of the risks impact and its likelihood to the Risk Classification Matrix.

ESMD has prescribed a template Scoring Scheme at the Directorate Level of the organization. This scheme defines four Impact Categories for which a risk may be scored. The Impact Categories are Safety, Performance, Cost and Schedule. Either qualitative or quantitative criteria may be used to score a risk and a Risk Scorecard is typically defined for each organizational level which defines that groups criteria for rating a risk against each of the four impact categories. Figure 32 shows the "Score Card" proposed for use by ESMD's Crew Exploration Vehicle (CEV) project.

Further risk analysis is prescribed in Ref. 2 in that the risks identified should be prioritized in a formal "Risk List". ARM will in automated fashion generate these Risk Lists based upon the Risk Scores assigned to each risk by virtue of its location in the Risk Classification Matrix. However a program manager typically desires greater control over this prioritization, and may desire to remove the ambiguity which will occur in a large program where multiple risks assigned to the same risk matrix cell will have the same Risk Score. Program Manager prioritization and reporting of a Risk's Priority along with its Score is a report which should be used to show this effect. Note also that the Risk Scores assigned by classification to a cell in Fig. 31 are not equal to a multiplication of the ordinal values for Probability and Impact. Rather the cell scores are assigned based upon a logic which in this implementation biases higher scores towards risk which are more affecting by their consequence than by their probability ranking. This scoring scheme represents a slight divergence over what NASA has used for other typical Risk Management activities $^{69-71}$ however it more clearly emphasizes the issue of biasing consequence as having a slightly greater effect on risk score than likelihood. 


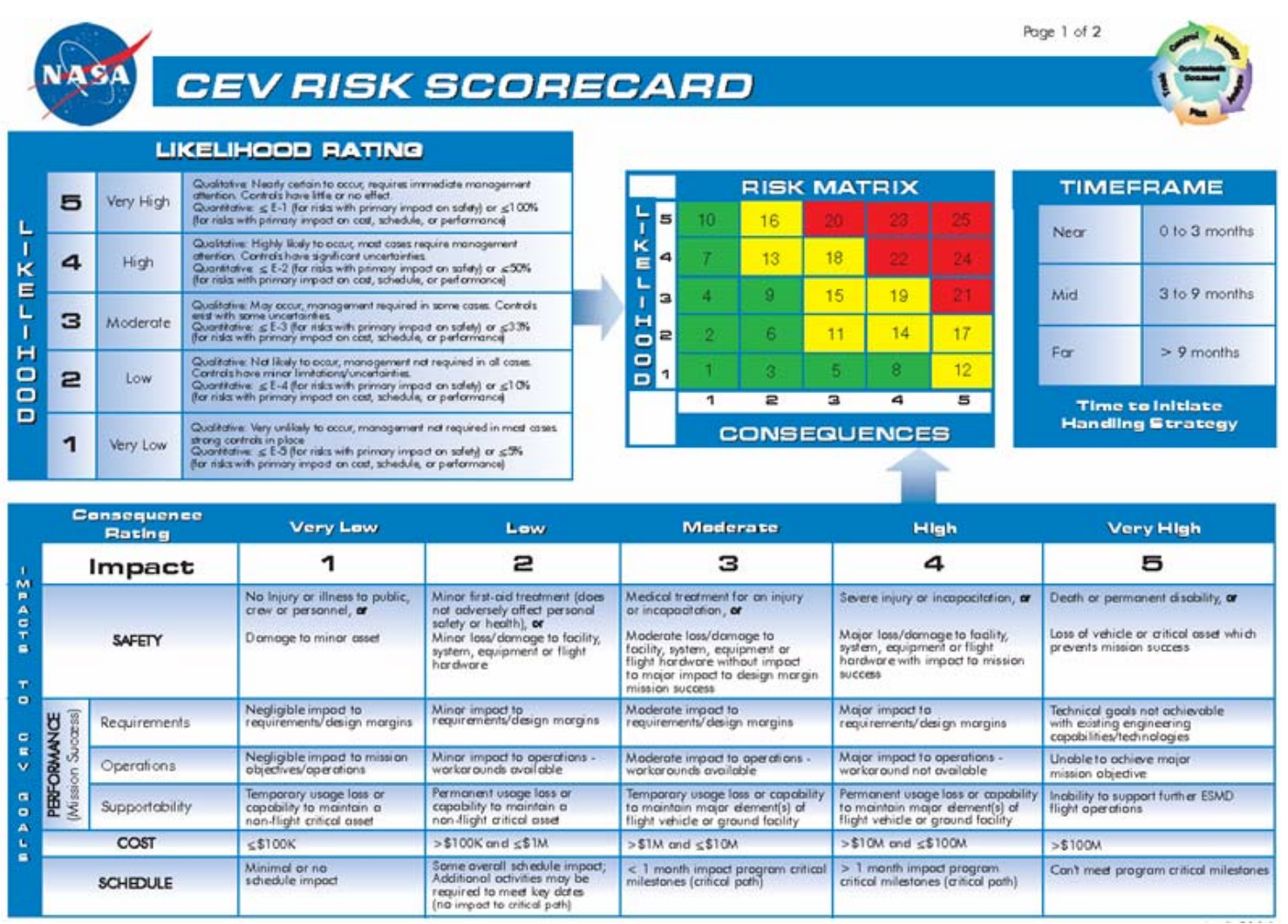

Figure 32 - CEV Scoring Scheme, A typical project level Scorecard

\section{B. Risk Management Process and Options:}

The ESMD Risk Management Process is structured from a "corporate model" where responsibility is held at the lowest possible effective organizational levels. Top level direction and distribution of resources flows down to the sub-tier organizations; so too does the Risk Management process. At the agency level, Refs. 1 and 2 prescribe Risk Management requirements for ESMD. At the next level the Directorate has created a Risk Management Plan where it prescribes the necessary process requirements to ensure proper sharing of data. Reporting and tool integration consistencies are part of these requirements. Below the Directorate level the Program level organizations create individual Risk Management Plans which suit their program management requirements and still adhere to the higher level requirements. Similarly the Project level organizations create a Risk Management Plan which includes Program, Directorate, and Agency prescriptions. For large programs/projects a formal risk review board may be configured and meet regularly to adjudicate risks. For smaller or less disparate activities the Risk Management activities may occur formally only as a part of regular program/project management meetings. Each organization in ESMD defines a Risk Management Officer (RMO) who may or may not be a person distinct from that element's Program/Project Manager. The RMO is responsible for assisting the PM and implementing the organizations CRM process. The RMO is also responsible for ensuring CRM/ARM training for personnel in his organization is supplied. Directorate level training is provided to all, but more specific functionalities and process requirements may be enforced at the lower organizational levels.

One of the most useful things a program manager can do to effectively utilize the CRM process is to track Technical Performance Measures, Key Performance Parameters, Figures of Merit and other such program measuring-sticks as risks. The International Council on Systems Engineering describes the advantages gained by keeping such data in a Risk Management system ${ }^{72}$.

As previously highlighted, Communication is a primary asset of CRM. For ESMD's CRM/ARM installation, communication of risk data to appropriate managerial levels is handled in three particular manners. First the database structure and access privileges as we have discussed promote sharing of risk data across the entire Directorate, its sub-tier organizations, and its supporting contracted service vendors. Secondly the reporting capability and a feature called "Alerts" enables users to use the ARM tool along with their Risk Review procedures to show risk data to other organizations either horizontally or vertically throughout the organization structure. Such 
alerts can be used for informational purposes as well as to document the needs for requesting additional risk mitigation resources. Finally if a particular organization desires that a risk they are in charge of be moved to another more appropriate responsible organizational element, this can be accomplished by a risk transfer. A risk transfer to a parent organizational element may be termed a Risk Escalation.

\section{Decision Methods}

Successful Continuous Risk Management requires sound decision making for risk identification, analysis, and control. The Logic-Evolved Decision (LED) analysis software framework is a modeling and decision support tool for performing risk-based analyses, assessments, and prioritizations for a broad range of applications. This top-down analysis approach uses software tools and accompanying methodology developed at the Los Alamos National Laboratory (LANL) for modeling the behavior of complex systems with respect to decision support applications. The LED computational algorithm uses linked, formal logic models to represent the basic functions of a decision analysis tool. This systems analysis capability incorporates fuzzy logic, approximate reasoning, possibility, probability, multi-attribute scoring, and graph theory to construct these decision support models. LED is a flexible, self-contained, comprehensive, and traceable decision support software tool for risk-based prioritization, hazard assessment, and portfolio management across a broad spectrum of applications.

The use of LED techniques has proven scalable, flexible, and reproducible for a wide range of decision support applications. The scalability of the LED analysis technique allows for detailed analysis of specific problems as well as a global tool for risk-based management of major and/or multiple systems. These techniques have been used in nuclear weapons safety applications, reducing espionage risk, evaluating sabotage risk, determining the risk of protected material theft, risk-based asset and infrastructure management, and evaluating risk-reduction measures for natural hazards including lightning and wildfire. The LED analysis approach and methodology has been thoroughly peer-reviewed by independent organizations including the American Society of Mechanical Engineers and the Monterey Institute for International Studies. The Monterey Institute's extensive review concluded that the LED approach is the most comprehensive counter-terrorism risk tool available.

The discussion in the Decision Methods section of this paper focuses on the collaborative effort between NASA, LANL, the National Institute of Aerospace, and Volpe Center Department of Transportation to develop risk-based assessments for the purpose of improving the overall security of the national Air Transportation System (ATS). The objective of these assessments is to provide a decision support tool that can be used to prioritize NASA research in aviation security based upon a comprehensive ATS risk assessment and other portfolio management attributes pertaining to technology development and implementation.

\section{NASA's Application of the LED Process: Risk-based Prioritization of NASA Research in Aviation Security}

Recently, LED techniques were used to identify, assess, and rank order the risks facing the United States ATS. An analysis of the threats facing the entire spectrum of aviation activities within the ATS was conducted in an effort to prioritize the NASA Aviation Safety and Security Program (AvSSP) security research portfolio. This LED portfolio prioritization process developed for the NASA AvSSP was based upon a comprehensive ATS risk assessment. Although many metrics are available for the technology prioritization process, such as cost, technical development issues, etc., the initial metric chosen for the technology rank ordering was the risk reduction associated with terrorist attacks on the ATS ${ }^{73}$. This top-down analysis effort assisted NASA in prioritizing its research in aviation security by providing NASA with a risk-based rank ordering of technology impact on national ATS terrorist attacks. As a result, this analysis and assessment effort yielded a two-fold purpose by accomplishing two objectives of national importance:

- A comprehensive ATS risk assessment based on terrorist attacks was developed for the nation.

- A decision support tool for the purposes of prioritizing NASA research in aviation security was developed.

For the purposes of this analysis, the concepts of combat aircraft survivability are used to examine terrorist risk $^{74}$. An attack scenario is a complete description of the process by which an adversary carries out an operation against a target. Risk is the expected loss and terrorist risk is evaluated for individual attack scenarios in this analysis. Total risk is determined from an aggregation over a set of scenarios for one or more targets of interest. Risk is expressed in terms of susceptibility, vulnerability, and consequences by the following relationship: 


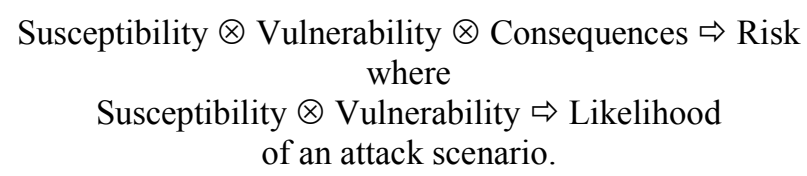

Translated to the problem being studied, security of the ATS, and linguistic expressions, susceptibility corresponds to the likelihood that an attack scenario is completed. Furthermore, susceptibility depends upon (1) the likelihood that the attacker chooses to attempt the attack (attractiveness), (2) the likelihood that the attack is carried to completion absent any intervention, and (3) the likelihood that the defender can prevent the attack from being completed. Vulnerability is the likelihood that the level of damage (the consequences given completion of the scenario) is such that the attack is considered to be successful by the attacker. Vulnerability depends upon (1) the intrinsic response of the target to the attack and (2) the capability of the defender to mitigate the consequences of the attack. Likelihood is thus a measure of the "belief" that an attack scenario will occur. Likelihood can be expressed in the following ways:

- Linguistic: "A is very unlikely"; "A is more likely than B"

- Frequentist: $\mathrm{f}(\mathrm{A})=2 \times 10^{-3} /$ year ; $\mathrm{f}(\mathrm{A})>\mathrm{f}(\mathrm{B})$

- Probabilistic: $\mathrm{p}(\mathrm{A})=.001 ; \mathrm{p}(\mathrm{A})>\mathrm{p}(\mathrm{B})$

- Odds: Payoff indifference between choices

Due to the lack of historical data concerning terrorist attacks against the ATS, the linguistic approach incorporating approximate reasoning is chosen to infer the likelihood and consequence of terrorist attacks against the ATS. The approximate reasoning approach incorporated in this LED based analysis and assessment uses expert judgment to infer the outcome, Likelihood $\otimes$ Consequences $\Rightarrow$ Risk, of terrorist attacks against the ATS.

The NASA AvSSP approach is to consider aviation security for the entire ATS. For analysis purposes, the entire ATS was therefore systematically divided into 3 distinct sub-systems: (1) aircraft, (2) airports, and (3) airspace. The aircraft sub-system was further decomposed into Federal Aviation Regulation (FAR) parts since the risk of a terrorist attack on the aircraft sub-system is dependent on the various aircraft operations. The aircraft sub-system decomposition included: (1) FAR Part 121 Passenger/Cargo-Outsider and Insider Attacks, (2) FAR Part 121 All Cargo, (3) FAR Part 135, and (4) FAR Part 91 aircraft operations.

After decomposing the ATS into the appropriate sub-systems, the first step in the risk assessment/technology prioritization process is to brainstorm the terrorist attack scenarios as shown in Figure 33. This scenario development

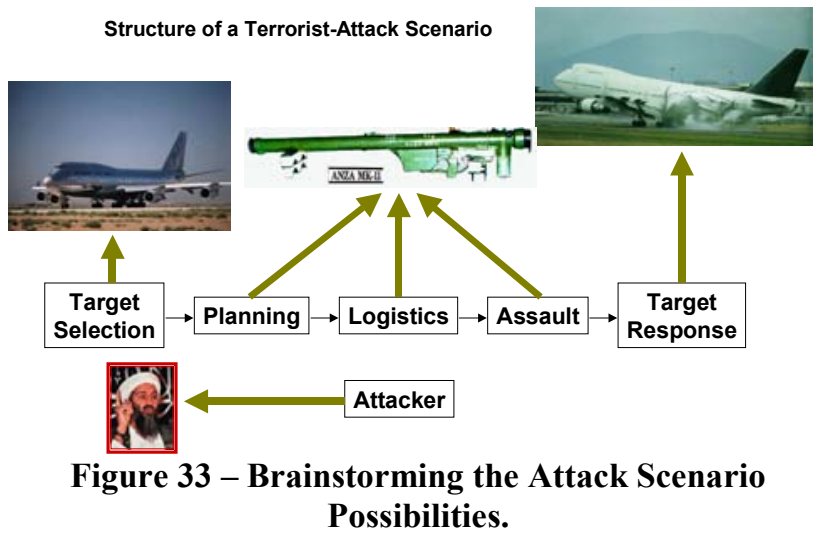

process involves methodically identifying threats facing the ATS and then determining the attacker/attacker group, target selection, planning, logistics, the actual assault, and the ultimate target response for each sub-system of the ATS. These elements are then rolled into detailed attack scenarios using a top-down approach for each ATS subsystem. Subject matter expert input is used in this attack scenario development step. The various subject matter experts used in this step include intelligence analysts, NASA aircraft/aircraft operations experts, aviation system expert consultants, pilots, airport managers, air traffic controllers, LANL decision support experts, and NASA security project researchers. 
The next step in the analysis process is to input the detailed attack scenarios for each ATS sub-system into the LEDTools software package. The LEDTools software is used to construct the attack scenarios in a hierarchal tree

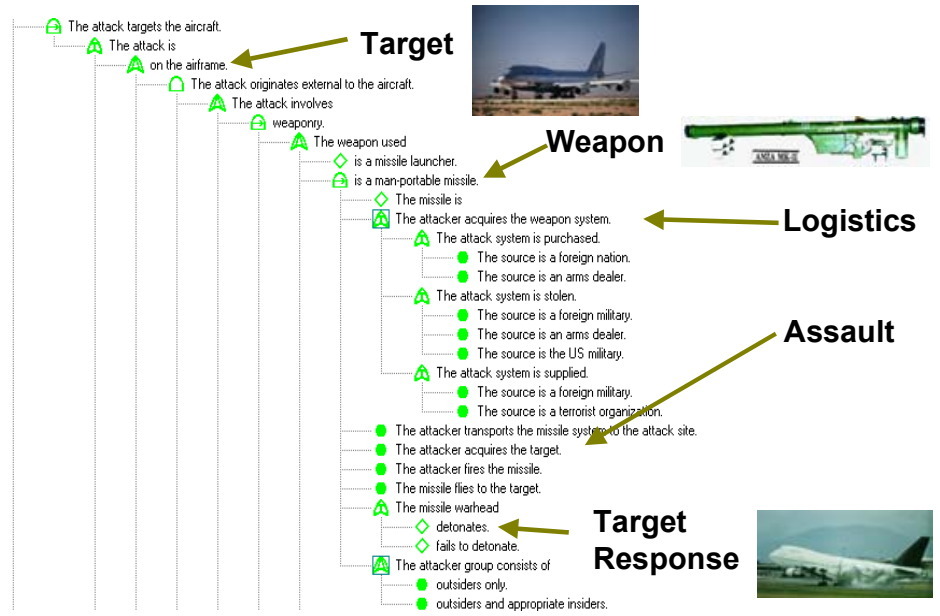

Figure 34 - Hierarchal Tree Structure of an Attack Scenario in LEDTools.

structure. The LEDTools software contains logical gates and operators that are used to construct the possibility trees as shown in Figure 34. Some of the logical gates within LEDTools include AND, Ordered AND, Combination, Inclusive OR, various Causal gates, Intersection, Union, Difference, Element, Taxonomy, and Process gates. These gates are used in conjunction with textual input to form the attack scenarios in a logical, hierarchal tree structure. The possibility trees can be solved in order to obtain a comprehensive, textual set of attack scenarios for each ATS sub-system. As a result, the paths of the possibility tree are indeed the attack scenarios. An example of one solution to the possibility tree shown in Fig. 34 is displayed below:

The attack is on the US aviation system. The attack is against the commercial aviation system. The targeted system is classified as a Part 121 air-carrier operation. The air-carrier operation handles passenger and cargo traffic. The attack targets the aircraft. The attack is on the airframe. The attack originates external to the aircraft. The attack involves weaponry. The weapon used is a man-portable missile. The attacker acquires the weapon system. The attacker transports the missile system to the attack site. The attacker acquires the target. The attacker fires the missile. The missile flies to the target. The missile warhead detonates. The attacker group consists of outsiders only.

This possibility tree solution capability of LEDTools is a very powerful functionality that aids in the attack scenario development process since this solution displays each scenario contained within the possibility tree in textual form. After all scenarios are developed for all of the possible attacks against the ATS, the scenarios are reduced to ATS element spanning sets. This is actually a screening process for developing a workable sub-set of scenarios that are representative of a larger class of attacks. This process is helpful since it is easier to deal with hundreds of scenarios rather than millions of scenarios. 
The third step in the analysis process is to develop the risk inferential models for the defender and attacker. The risk inferential models include attack scenario susceptibility, vulnerability, and consequence. These models also include the baseline risk as it exists today for the ATS with the current preventive and mitigating measures in place and various attacker intent sub-models. The logical tree structure of the risk inferential model is displayed in Fig. 35 and the various computational elements that comprise the risk inferential model are shown. The approximate reasoning approach used in this study utilizes subject matter expert elicitation to populate the variables within the risk models. The various subject matter experts used in this step include intelligence analysts, NASA

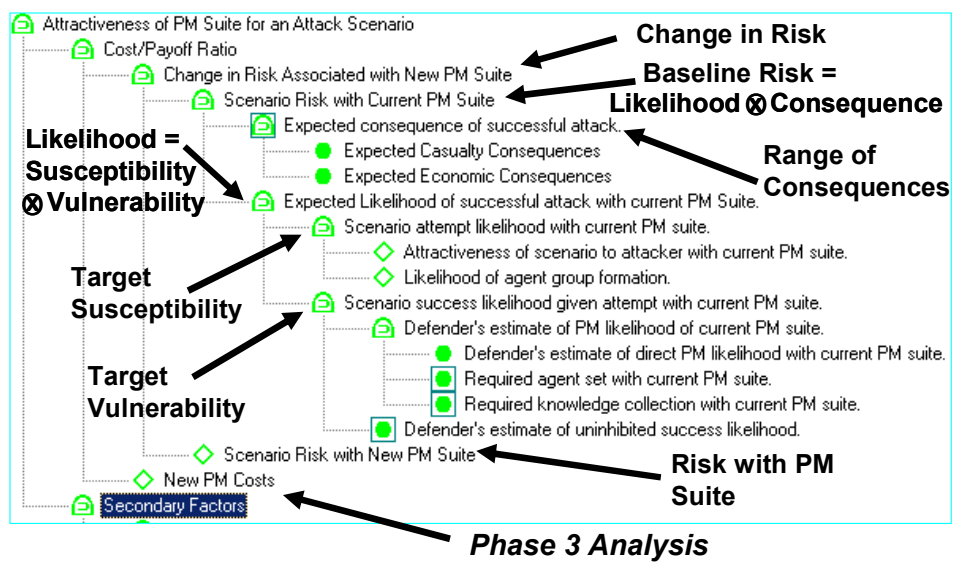

Figure 35 - Risk Inferential Model for the Defender and Attacker with Call-out for Various Risk Model Components.

aircraft/aircraft operations experts, aviation system expert consultants, pilots, airport managers, air traffic controllers, LANL decision support experts, nuclear and chemical/biological expertise, electromagnetic effects expertise, cost expertise, and NASA security project researchers.

We are now capable to determine and identify the attack scenario baseline risk for each ATS sub-system which includes [the aircraft] FAR Part 121 Passenger/Cargo-Outsider and Insider Attacks, FAR Part 121 All Cargo, FAR Part 135, FAR Part 91, the airport, and the airspace. At this point in the analysis process, the first objective for this overall systems analysis effort of developing a comprehensive ATS risk assessment based on terrorist attacks for the nation is accomplished. An illustrative example of attack scenario baseline risk output for a specific ATS subsystem is shown below in Figure 36.

Dependence of Scenario Risk on Attacker Type

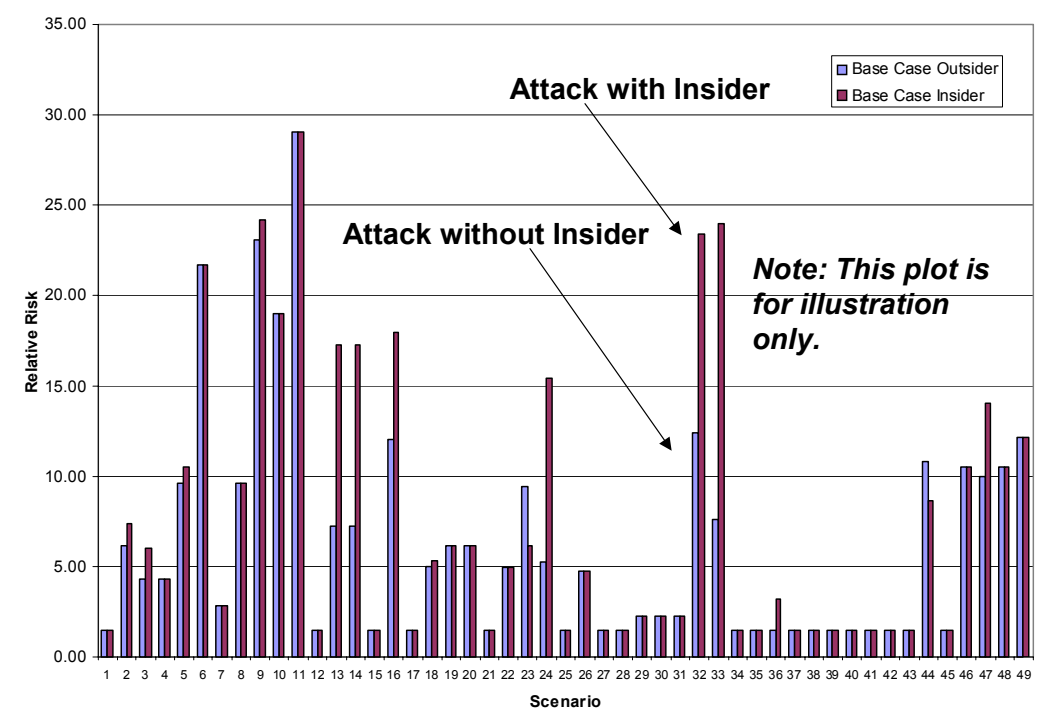

Figure 36 - Illustrative Example of Attack Scenario Baseline Risk for ATS Sub-system. 
Step 5 in this analysis process involves mapping and applying the NASA technologies, which are combinations of preventive and mitigating (PM) measures, to the attack scenarios in order to determine technology risk reduction potential based on terrorist attacks. Step 5 is summarized in Figure 37. The technologies under development are evaluated for three levels of integration to determine technology risk reduction potential and prioritization. The three different categories of technology integration are:

- Stand-Alone

- Integrated

- Enhanced Integration

The first analysis sub-set looks at each of the technologies operating independently in a stand-alone capability. This base case risk reduction potential does not consider how the technologies would work in an integrated fashion. The second analysis category

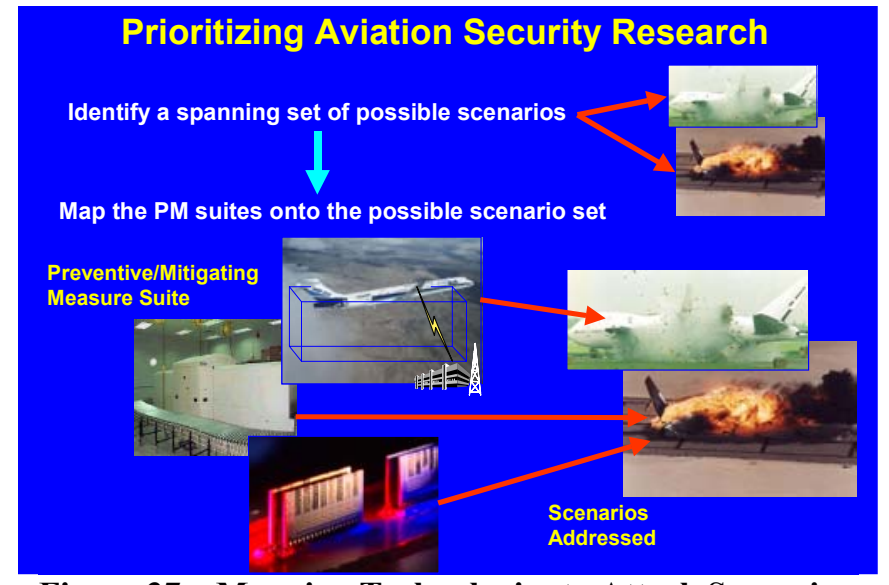

Figure 37 - Mapping Technologies to Attack Scenarios. is for the integrated Concept of Operations (CONOPS) case where the natural synergies of the technologies are considered in a concept of operation. In this CONOPS case, the technologies are assessed based on the current AvSSP project plan level of integration and levels of effort. In developing the CONOPS and considering the scenarios and threats, technology gaps are exposed. This natural exposure of gaps resulting from the logical flow of the models is another benefit of the LED methodology. Extra capabilities needed from the technologies to fill these gaps are determined. In this study, a new notional product, deemed the Collaborative Security Integrator (CSI), is developed. The CSI is the third category of integration and allows for the necessary data sharing and communication links plus additional attributes that are required to achieve even greater risk reduction potential from the technologies. The CSI category is defined as the enhanced technology integration capability for expressing terrorist attack risk reduction potential.

The final step of this analysis effort is to evaluate the technology attack scenario risk reduction potential for the three levels of integration described above. The analysis results provide the NASA AvSSP with research portfolio prioritization recommendations based on attack scenario risk reduction. The risk reduction potential is defined as the difference between the ATS baseline risk (as it exists today) and the new terrorist attack scenario risk based on technology insertion. The analysis results provide the capability to rank order the risk reduction potential of applicable NASA research technologies. At this point in the analysis process, the second and final objective for this overall systems analysis effort of developing a decision support tool for the purposes of prioritizing NASA research in aviation security is accomplished. The ideal technology risk reduction potential and NASA AvSSP technology portfolio prioritization methodology for the three levels of integration is shown below in Figure 38. This risk reduction potential is termed ideal since it is exclusively based upon terrorist attack risk reduction. The technology nomenclature has been removed from the $\mathrm{x}$-axis due to security classification in Figure 38. 


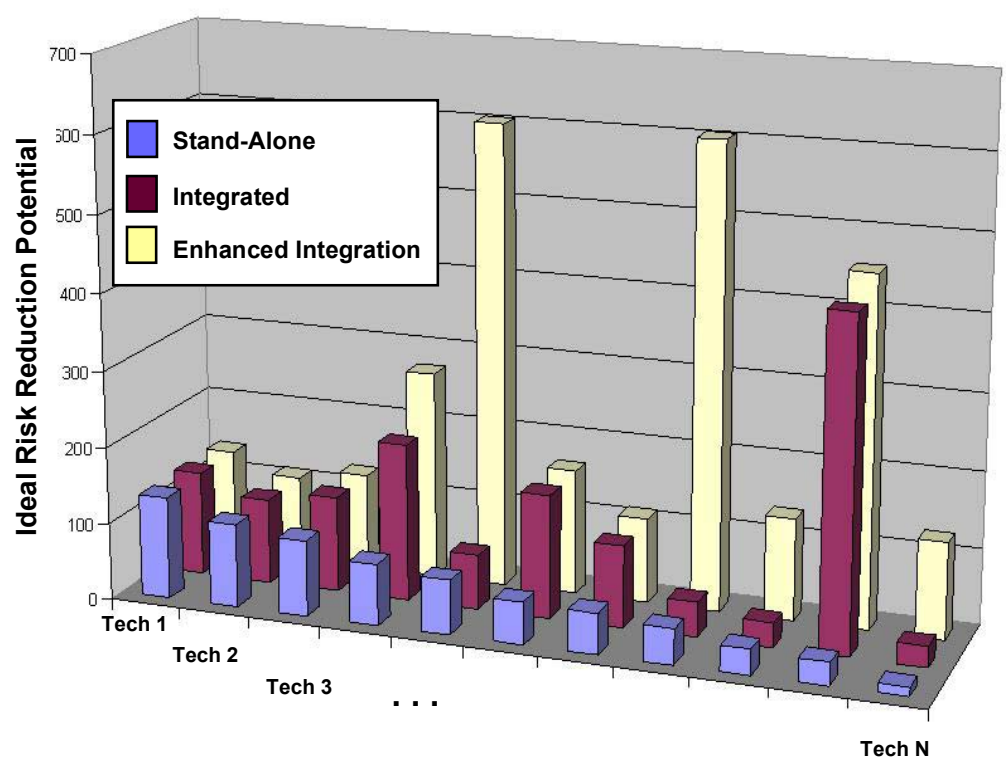

Figure 38 - Ideal Technology Risk Reduction Potential for the Three Categories and NASA AvSSP Technology Portfolio Prioritization.

\section{Conclusions}

The NASA LaRC Systems Analysis and Concepts Directorate (SACD) includes a wide variety of capabilities, tools, methods, and experience areas of uncertainty quantification, propagation, decomposition, and management, risk analysis, robust design, reliability methods, and logic-evolved decision analysis within SACD. This group of disciplines referred to herein as Decision Support (DS) methods and tools, have been, or are currently being widely used within a broad spectrum of SACD applications. The DS disciplines are distinct from, and span across, the other technical disciplines typically used within SA such as structures, fluid and thermal dynamics, cost estimation, and reliability analysis. The DS methods and tools fill three critical roles in the SA process: 1) they provide the evaluations of uncertainty, risk, decision-making metrics within the analysis or design process, and 2) they provide feedback to the system analysis and design processes which helps to shape, define, and constrain the feasible analysis or design space and, perhaps, even the analysis and design paths used, and 3) they provide feedback to decision makers or stakeholders managing the analysis or design process so they can make informed choices. Many applications of these techniques were illustrated in this paper.

\section{References}

1"NASA Program and Project Management Processes and Requirements," NASA Procedural Requirements, NPR 7120.5C, URL: http://nodis3.gsfc.nasa.gov/displayDir.cfm?Internal_ID=N_PR_7120 005C_\&page_name=main, Effective Date: March 22, 2005 [cited 10 Jul 2006].

2"'Risk Management Procedural Requirements w/Change 1 (4/13/04)," NASA Procedural Requirements, NPR 8000.4, URL: http://nodis3.gsfc.nasa.gov/displayDir.cfm?Internal_ID=N_PR_8000_0004_\&page_name=main, Effective Date: April 25, 2002 [cited 10 Jul 2006].

3“"Probabilistic Risk Assessment (PRA) Procedures for NASA Programs and Projects," NASA Procedural Requirements, NPR 8705.5, URL: http://nodis3.gsfc.nasa.gov/displayDir.cfm?Internal_ID=N_PR $8705 \quad 0005$ \&page name=main, Effective Date: July 12, 2004 [cited 10 Jul 2006].

${ }^{4}$ Cavanaugh, S., Kumar, A., Rausch, V., Brewer, L., Kimmel, W., and Korte, J., "Overview of NASA Langley’s Systems Analysis Capabilities," AIAA-2006-7025, Sep. 2006.

"Systems Engineering Procedural Requirements," NASA Procedural Requirements, NPR 7123.1, URL: http://nodis3.gsfc.nasa.gov/displayDir.cfm?Internal_ID=N_PR_7123 0001_\&page_name=main, Effective Date: March 13, 2006 [cited 10 Jul 2006]. 
${ }^{6}$ Chytka, T., Brown, R., Shih, A., and Reeves, J., “An Integrated Approach to Life Cycle Analysis,” AIAA-20067027, Sep. 2006.

${ }^{7}$ Columbia Accident Investigation Board, Columbia Accident Investigation Board Report, Volume 1, U. S. Government Printing Office, URL: http://caib.nasa.gov/news/report/pdf/vol1/full/caib_report_volume1.pdf, August 2003.

${ }^{8}$ Return to Flight Task Group, Return to Flight Task Group Final Report, U. S. Government Printing Office, URL: http://returntoflight.org/assets/pdf/final_rtftg_report/RTF_TG_Final_Report_Lo-Res.pdf, July 2005.

${ }^{9}$ Green, L.; Newman, P.; and Haigler, K., "Sensitivity Derivatives for Advanced CFD Algorithm and Viscous Modelling Parameters Via Automatic Differentiation," AIAA 93-3321, 1993. Presented at the 11th AIAA Computational Fluid Dynamics Conference, Orlando, FL, July 6-9, 1993.

10 Zang, T. A.; and Green, L. L., "Multidisciplinary Design Optimization Techniques: Implications and Opportunities for Fluid Dynamics Research," AIAA 99-3798. Presented at the 30th AIAA Fluid Dynamics Conference, Norfolk, Virginia, June 28-July 1, 1999.

11 Putko, M.; Newman, P. A.; Taylor A. C. III; and Green, L. L., "Approach for Uncertainty Propagation and Robust Design in CFD Using Sensitivity Derivatives," AIAA 2001-2528. Presented at the 15th AIAA Computational Fluid Dynamics Conference, Anaheim, California, June 11-14, 2001.

12 Green, L. L.; Lin, H. Z.; and Khalessi, M. R., "Probabilistic Methods for Uncertainty Propagation Applied to Aircraft Design," AIAA 2002-3140. Presented at the 20th AIAA Applied Aerodynamics Conference, St. Louis, MO, June 24-26, 2002.

13 Green, L. L.; and Spence, A. M., "Applications of Computational Methods for Dynamics Stability and Controls," AIAA 2004-0377. Presented at the 42nd AIAA Applied Aerospace Sciences Conference and Exhibit, Reno, NV, January 5-8, 2004.

14 Zang, T. A., Hemsch, M. J., Hilburger, M. W., Kenny, S. P., Luckring, J. M., Maghami, P., Padula, S. L., and Stroud, W. J., "Needs and Opportunities for Uncertainty-Based Multidisciplinary Design Methods for Aerospace Vehicles," NASA Langley Research Center NASA/TM-2002-211462.

15 Alben, K. T., "Design, Analyze, and Optimize with Design-Expert," Analytical Chemistry, Volume 74, (7), Pages 222 A-223 A., April 2002.

16 Dentener, A., "Design-Expert DOEs it Better," Food Technology in New Zealand Magazine, URL: http://www.foodtechnology.co.nz, Volume 37 (9): pp. 12-14, September 2002.

17 Goodliff, K., Cornelius, D., and Sasamoto, W., "Stochastic Orbital Lifetime Analysis," Paper Number SSC06-VIII-6, From 20th Annual AIAA/USU Conference on Small Satellites, Aug. 2006.

18 Johnson, R., "Elementary Statistics," Duxbury Press, New York, 1996.

19 Anderson, M., and Whitcomb, P., "DOE Simplified: Practical Tools for Effective Experimentation," Productivity Press, ISBN: 1563272253, January 1999.

20 Anderson, M., and Whitcomb, P., "RSM Simplified: Optimizing Processes Using Response Surface Methods for Design of Experiments," Productivity Press, ISBN: 1563272970, 292 pages Published January 2005.

${ }^{21}$ Cruz, J., and S. G. Anders: "Assessment of an Unstructured-Grid Method for Predicting Aerodynamic Performance of Jet Flaps," AIAA 2006-3868, June 2006.

22 Bischof, C., Carle, A., Corliss, G., Griewank, A., and Hovland, P., "ADIFOR - Generating Derivative Codes from Fortran Programs," CRPC-TR91185 or ANL/MCS-P263-0991 December, 1991. Also appears in Scientific Programming, no. 1 (1992), pp. 1-29.

23 Bischof, C., Carle, A., Khademi, P., and Mauer, A., "The ADIFOR 2.0 System for the Automatic Differentiation of Fortran 77 Programs," CRPC-TR94491 or ANL/MCS-P481-1194, 1994.

24 Bischof, C., Carle, A., Hovland, P., Khademi, P., and Mauer, A., "ADIFOR 2.0 User's Guide," CRPCTR95516-S or ANL/MCS-TM-192, March, 1995. Revised: May, 1995.

25 Bischof, C.; Corliss, G.; Green, L.; Griewank, A.; Haigler, K.; and Newman, P., "Automatic Differentiation of Advanced CFD Codes for Multidisciplinary Design," Presented at Symposium on High Performance Computing for Flight Vehicles, Arlington, VA, December 7-9, 1992.

${ }_{26}$ Green, L.L.; Bischof, C.; Carle, A; Griewank, A; Haigler, K.; and Newman, P., "Automatic Differentiation of Advanced CFD Codes With Respect to Wing Geometry Parameters for MDO," Abstracts from the Second U.S. National Congress on Computational Mechanics, Washington, D.C., Aug. 16-18, 1993, p. 136.

27 Carle, A.; Green, L.; Bischof, C.; and Newman, P., "Applications of Automatic Differentiation in CFD," AIAA 94-2197, 1994. Presented at the 25th AIAA Fluid Dynamics Conference, Colorado Springs, CO June 20-23, 1994. 
28 Ashby, D. L., Dudley, M. R., Iguchi, S. K., Browne, L., and Katz, J., "Potential Flow Theory and Operation Guide for the Panel Code PMARC," NASA TM 102851, January 1991. (Unofficially revised in 1999 for PMARC version 14).

${ }^{29}$ Klein, V., Murphy, P. C., Curry, T. J., and Brandon, J. M., "Analysis of Wind Tunnel Longitudinal Static and Oscillatory Data of the F-16XL Aircraft," NASA/TM-97-206276.

${ }^{30}$ Kirby, M., Burg, C., Tai, J., "Technology Solicitation and "Bottoms Up" Assessment Subsonic Transport (ST) Sector VISTA -Deliverable II," PowerPoint Presentation.

31 Kirby, M., Mavris, D., Largent, M., "A Process for Tracking and Assessing Emerging Technology Development Programs for Resource Allocation," AIAA 2001-5280.

32 Kirby, M., Mavris, D., "Forecasting Technology Uncertainty in Preliminary Aircraft Design," SAE-AIAA1999-01-5631.

33 Kirby, M., "TIES for Beginners," 3rd Edition. August 2002.

34 Kenny, S., Giesy, D., Crespo, L., Burg, C., Zang, T., Green, L., Chwalowski, P., "Confidence Module of CDS," CDS Day. PowerPoint Presentation. October 2005.

35 Batson, R.G., Love, R.M., "Risk Analysis Approach to Transport Aircraft Technology Assessment," Journal of Aircraft, Vol. 25, No 2, February 1988, pp 99-105.

36 Carle, A., Fagan, M., and Green, L., "Preliminary Results from the Application of Automated Adjoint Code Generation to CFL3D," AIAA98-4807. Presented at the 7th AIAA/USAF/NASA/ISSMO Symposium on Multidisciplinary Analysis and Optimization, St. Louis, Missouri, September 2-4, 1998.

37 Green, L., Carle, A., and Fagan, M, "Demonstration of Automatically-Generated Adjoint Code for Use in Aerodynamic Shape Optimization," Presented at the HPCCP/CAS Workshop, August 24-26, 1998. NASA/CP1999-208757 - 1998 HPCCP/CAS Workshop Proceedings, January 1999.

38 Biedron, R. T., Samareh, J. A., and Green, L. L., "Parallel Computation of Sensitivity Derivatives with Application to Aerodynamic Optimization of a Wing," Presented at the HPCCP/CAS Workshop, August 24-26, 1998. NASA/CP-1999-208757 - 1998 HPCCP/CAS Workshop Proceedings, January 1999.

39 Park, M. A., and Green, L. L., "Steady-State Computation of Constant Rotational Rate Dynamic Stability Derivatives," AIAA 2000-4321. Presented at the 18th AIAA Applied Aerodynamics Conference, Denver, Colorado, August 14-17, 2000.

40 Raney, D. L., Montgomery, R. C., Green, L. L., and Park, M. A., "Flight Control Using Distributed ShapeChange Effector Arrays," AIAA 2000-1560. Presented at the 41st AIAA/ASME/ASCE/AHS/ASC Structures, Structural Dynamics, and Materials Conference and Exhibit, Atlanta, Georgia, April 3-6, 2000.

${ }^{41}$ Park, M. A., Green, L. L., Montgomery, R. C., and Raney, D. L., "Determination of Stability and Control Derivatives Using Computational Fluid Dynamics and Automatic Differentiation," AIAA 99-3136. Presented at the 17th AIAA Applied Aerodynamics Conference, Norfolk, Virginia, June 28-July 1, 1999.

42 Luckring, J. M., Hemsch, M. J., Morrison, J. H., "Uncertainty in Computational Aerodynamics," AIAA2003-0409, 2003.

${ }^{43}$ Sobieszczanski-Sobieski, J., Haftka, R.T., "Multidisciplinary aerospace design optimization: Survey of recent developments," Structural Optimization, Vol. 14, No. 1, August 1997, pp. 1-23.

44 Alexandrov, N.M., Hussaini, M.Y., "Multidisciplinary Design Optimization: State of the Art," Society for Industrial and Applied Mathematics, Philadelphia, 1997.

45 Alexandrov, N.M., Lewis, R.M., "Analytical and Computational Aspects of Collaborative Optimization," NASA/TM--210104-2000, April 2000.

46 Alexandrov, N.M., Lewis, R.M., “Analytical and Computational Properties of Collaborative Optimization for Multidisciplinary Design,” AIAA Journal, Vol. 40, No. 2, February 2002, pp. 301-309.

${ }_{47}$ Alexandrov, N.M., Lewis, R.M., "Analytical and computational properties of distributed approaches to MDO,” AIAA-2000-4718, September 2000.

48 Alexandrov, N.M., Lewis, R.M., "Algorithmic perspectives on problem formulations in MDO," AIAA-20004719, September 2000

49 Alexandrov, N.M., Lewis, R.M., "Dynamically Reconfigurable Approach to Multidisciplinary Problems," AIAA-2003-3431, June 2003.

50 Alexandrov, N.M., Lewis, R.M., "Reconfigurability in MDO Problem Synthesis, Part 1 and Part 2," Papers AIAA-2004-4307 and AIAA-2004-4308, August 2004.

51 Chiralaksanakul, A., Mahadevan, S., "First-Order Methods for Reliability-Based Optimization," ASME Journal of Mechanical Design, Vol.127, No. 5, pp. 851-857, 2005.

52 Smith, N., Mahadevan, S., "Integrating System-Level and Component-Level Designs Under Uncertainty," Journal of Spacecraft and Rockets, 42 (2005), 752-760. 
53 Smith, N., Mahadevan, S., Probabilistic Methods for Aerospace System Conceptual Design," Journal of Spacecraft and Rockets, 40 (2003), 411-418.

54 Smith, N., "Probabilistic Methods for Multidisciplinary System Design," M.S. Thesis, Vanderbilt University, 2002.

55 Mahadevan, S., Smith, N., "System Risk Assessment and Allocation in Conceptual Design," NASA CR2003-212162, May 2003.

56 Alexandrov, N. M., Dennis, J. E., Lewis, R. M., Torczon, V., “A Trust Region Framework for Managing Use of Approximation Models in Optimization," Journal on Structural Optimization, Vol. 15, No. 1, pp. 16-23, 1998.

57 Alexandrov, N. M., Nielsen, E.J., Lewis, R. M., Anderson, W. K., "First-Order Model Management with Variable-Fidelity Physics Applied to Multi-Element Airfoil Optimization," AIAA Paper 2000-4886, September 2000 .

58 Alexandrov, N. M., Lewis, R. M., Gumbert, C. R., Green, L. L., and Newman, P. A., "Optimization with variable-fidelity models applied to wing design," AIAA J. of Aircraft, Vol. 38, No. 6, pp.1093-1101, NovemberDecember 2001.

59 Alexandrov, N.M. and Lewis, R.M., "An Overview of First-Order Model Management for Engineering Optimization," Optimization and Engineering, Vol. 2, No.4, pp. 413-430, December 2001, published 2002.

60 Alexandrov, N.M. and Lewis, R.M., "First-Order Approximation and Model Management in Optimization," in "Large-Scale PDE-Constrained Optimization", Series: Lecture Notes in Computational Science and Engineering, Vol. 30, pp. 65-81, Biegler, L.T.; Ghattas, O.; Heinkenschloss, M.; Bloemen Waanders, B. V. (Eds), SpringerVerlag, 2003.

${ }_{61}$ Anderson, W. K., and Bonhaus, D. L., "Aerodynamic Design on Unstructured Grids for Turbulent Flows," Technical Report NASA-TM-112867, NASA Langley Research Center, 1997.

62 M.W. Trosset, M.W., N.M. Alexandrov, N.M., L.T. Watson, L.T., "New Methods for Robust Design Using Computer Simulation," Proceedings of the Section on Physical and Engineering Sciences, American Statistical Association, 2003.

${ }_{63}$ Gumbert, C. Hou, G., Newman, P., "High-Fidelity Computational Optimization for 3-D Flexible Wings: Part I - Simultaneous Aero-Structural Design Optimization (SASDO)," Optimization and Engineering, Vol. 6, pp117138, Springer, 2005.

64 Gumbert, C., Newman, P., Hou, G., "High-Fidelity Computational Optimization for 3-D Flexible Wings: Part II - Effect of Random Geometric Uncertainty on Design," Optimization and Engineering, Vol. 6, pp139-156, Springer, 2005.

${ }_{65}$ Gumbert, C., Hou, G. Newman, P., "Reliability Assessment of a Robust Design Under Uncertainty for a 3-D Flexible Wing," AIAA 2003-4094, 16th CFD conference, Orlando, FL, June 23-26, 2003.

${ }^{66}$ Strategic Thought Limited, Active Risk Manager, Commercial Software Application, URL: http://www.strategicthought.com.

67 Structured Software Systems Ltd (3SL), Cradle-5, Commercial Software Application, URL: http://www.threesl.com.

${ }_{68}$ Primavera Systems Incorporated, Primavera, Commercial Software Application, URL: http://www.primavera.com.

69 International Space Station Program Risk Management Plan - SSP 50175 Revision A, April 102002.

National Aeronautics and Space Administration, International Space Station Program Johnson Space Center,

Houston, Texas, Contract No. NAS15-10000

(http://pbma.hq.nasa.gov/docs/public/pbma/bestpractices/bp jsc 39.pdf).

70 Draft Space Shuttle Program Risk Management Plan, NSTS 07700 Revision O, National Aeronautics and Space Administration, Lyndon B. Johnson Space Center Houston, Texas 77058 July 22, 2005. (Currently not for public release).

71 Armstrong M.S., "Risk Management Plan for the EMD Project (ECS Maintenance and Development)," December 2003, Raytheon Company 1616 McCormick Drive Upper Marlboro, Maryland 20774-5301, Prepared Under Contract NAS5-03098 CDRL Item \#006. 206-EMD-001. (http://edhs1.gsfc.nasa.gov/waisdata/emd/pdf/206emd001.pdf).

72 Roedler G.J., Jones C., “Technical Measurement a Collaborative Product of PSM,” INCOSE and Industry, 27-December 2005, INCOSE TP-2003-020-01. (http://www.incose.org/ProductsPubs/pdf/TechMeasurementGuide_2005-1227.pdf)

73 Eisenhawer, S.W., Bott, T.F., Sorokach, M.R., Jones, F.P., Foggia, J.R., "Risk-Based Prioritization of Research for Aviation Security Using Logic-Evolved Decision Analysis," $22^{\text {nd }}$ International System Safety Conference, Conference Proceedings, ISBN: 0-9721385-4-4, pp. 106-115, August 2004. Refer to the following 
website for a complete listing of LANL authored publications discussing the LED analysis methodology and applications: http://www.lanl.gov/source/orgs/d/d5/publications.shtml.

74 Ball, R. "The Fundamentals of Aircraft Combat Survivability Analysis and Design," AIAA, 1985. 